\title{
SOBRE EL CALENDARIO \\ REPRODUCTIVO DE LAS MUJERES \\ ESPAÑOLAS
}

\section{Miguel Requena}

Universidad Nacional de Educación a Distancia

\section{RESUMEN}

El presente artículo tiene por objeto reconstruir algunas de las magnitudes básicas que han definido a lo largo del siglo el ciclo vital de la familia española, en particular las que se refieren al período reproductivo. La información de base procede de la Encuesta Sociodemográfica del Instituto Nacional de Estadística (1991), que ha sido sometida a una explotación específica con objeto de analizar en detalle el tempo de la reproducción de diversas generaciones de mujeres españolas. Las medidas básicas se refieren a la edad al nacimiento de los hijos y a los intervalos fecundos. La observación longitudinal de los datos permite medir el proceso de contracción del intervalo genésico que acompaña a la reducción de la descendencia final de las generaciones estudiadas y en el que tiene un peso decisivo el adelanto de la edad a la que se tiene el último hijo. Asimismo y con objeto de contrastar la fiabilidad de la fuente de datos utilizada, se realiza también un análisis transversal de la fecundidad instantánea que corresponde al momento de la encuesta.

\section{INTRODUCCION}

A lo largo del presente siglo el régimen demográfico español ha terminado por ajustarse a un modelo posmalthusiano de regulación de la población. Como se sabe, tal modelo se caracteriza por sus bajos niveles reproductivos en respuesta a la acusada prolongación de la vida humana que tiene lugar cuando 
se experimenta una brusca caída de las tasas de mortalidad como la que caracteriza a las modernas sociedades industriales. Es cierto que el comienzo relativamente simultáneo de las caídas de la mortalidad y la natalidad ha dado su tono peculiar a la transición española. Es cierto también que el retraso con que comenzó la caída de la mortalidad, y el rumbo paralelo y descendente que siguieron mortalidad y natalidad, nunca desencadenaron en España nada parecido a un estallido demográfico, sino que más bien provocaron un crecimiento sostenido, continuo y regular (Arango, 1987). Y que, debido a ese peculiar calendario, la caída de la mortalidad y la estabilización de la natalidad se han dado al final de la transición y no al principio, como ha sido la norma en otros países europeos de comportamientos demográficos más precoces (Tortella, 1994). A pesar de todo ello, la evolución secular ha terminado por instalarnos en un régimen reproductivo plenamente moderno. La sociedad española, en la medida en que se puede hablar de ella como un agregado que se comporta coherentemente, ha culminado a lo largo del presente siglo su particular transición demográfica. Tras casi ochenta años de fuerte crecimiento demográfico, la tasa interanual correspondiente a la década de los años ochenta $(0,47$ por 100$)$ está muy próxima a la que se produjo en la última década del siglo pasado $(0,44$ por 100$)$, momento en el que se puede establecer que comienza la transición de la mortalidad española.

Difícilmente el cambio del sistema de control de la población podría haber dejado intacta la institución humana en la que se construye socialmente el proceso de la reproducción biológica. Hay que tener en cuenta que, siendo la institución familiar la matriz social en la que se desarrolla ese proceso de reducción de la fecundidad, la transición demográfica es en muy buena medida un cambio trascendental de pautas familiares ${ }^{1}$. Una vez que decrecen las exigencias colectivas respecto de la fecundidad, las necesidades de reproducción de cada familia también disminuyen — bien que los mecanismos que explican esa disminución a escala de los individuos siguen siendo objeto de intensa controversia- El número total de hijos producidos por cada unidad familiar desciende, pero, además, la aparición de métodos anticonceptivos cada vez más potentes, cómodos y accesibles permite concentrar la procreación y crianza en períodos de tiempo más cortos. Los efectos de esta nueva situación son múltiples, sobre todo en la medida en que liberan una energía que antes se dirigía

1 De hecho, muy pronto se tuvo conciencia de que las implicaciones familiares de la revolución demográfica no se harían esperar. A partir de los años treinta del presente siglo — precisamente cuando la transición de la fecundidad camina ya con paso seguro en gran parte del área europea- aparecen los primeros análisis demográficos del nuevo sistema de familias pequeñas al que da lugar la posibilidad de mantener los niveles reproductivos engendrando menos hijos. La conciencia de las implicaciones familiares de las nuevas pautas demográficas se superpuso a la representacion del declive familiar que se venía extendiendo desde el siglo XIX. A lo largo del siglo pasado abundan, en efecto, los testimonios alarmados y los lamentos ideológicos ante lo que se suponía una decadencia de la familia de magnitud y trascendencia incalculables (Goldthorpe, 1987: 38). 
exclusiva o prioritariamente a la reproducción. Así, bajo las nuevas condiciones demográficas existe la posibilidad de dedicar a actividades extrafamiliares una cierta cantidad de recursos antes consagrada de forma principal a las labores que exige la fecundidad (Garrido, 1996).

De entre los recursos liberados de la carga de la reproducción tal vez sea el tiempo el que, por el alcance y la cantidad de sus consecuencias, merece citarse en primer lugar. La transición a un régimen demográfico moderno por fuerza ha de suponer un ahorro de tiempo reproductivo que introduce modificaciones sustanciales en el calendario biográfico de individuos y familias. Una manera de aproximarse a esos cambios es mediante el enfoque del ciclo vital de la familia, que goza ya de una larga ejecutoria en el campo de los estudios familiares desde los trabajos pioneros de Glick a finales de los años cuarenta (1947). La idea que subyace a este enfoque es en principio bastante simple: amalgamar en una única y simple estructura analítica los comportamientos demográficos de la fecundidad, la nupcialidad y la mortalidad. Puesto que la mayoría de los fenómenos demográficos básicos tienen lugar en el ámbito de la familia, se establecen las distintas fases del ciclo familiar y su duración a partir de esos acontecimientos que puntúan tan decisivamente las trayectorias vitales. Debido tanto a los comportamientos que incluye como a su afán por determinar la importancia relativa de cada una de las fases, el enfoque del ciclo vital se ajusta bastante bien al intento de evaluar la redefinición de las actividades familiares que resulta de los cambios en el modelo reproductivo.

Uno de los más señalados beneficios que reporta la utilización del enfoque del ciclo vital es, en efecto, el de situar en su contexto familiar los comportamientos vitales que constituyen los parámetros básicos de todo sistema demográfico. En cambio, el incoveniente más obvio es que, en su intento por sintetizar el propio concepto de ciclo vital familiar en una única estructura de análisis, puede llegar a reducir engañosamente la variedad real de trayectorias familiares que alberga una sociedad ${ }^{2}$. Si los puntos de comienzo y fin de las fases se establecen, por ejemplo, mediante alguna medida estadística de tendencia central como la media o la mediana, el resultado final puede llegar a ocultar las trayectorias familiares desviantes. Semejante problema se plantea, fundamentalmente, cuando se reduce todo posible ciclo a las trayectorias típicas de las familias nucleares, lo que muy a menudo sucede simplemente porque se opera en la práctica con la restricción de que los matrimonios son estables o de que la transición desde la familia de orientación a la de procreación es continua y lineal. Para eliminar los inconvenientes derivados de esa reducción y esas restricciones no hay más remedio que ampliar el enfoque y admitir la posibilidad de diferentes ciclos familiares dentro de una misma población (Höhn, 1987).

Es claro, por lo tanto, que los inconvenientes del enfoque del ciclo familiar son una función directa de la heterogeneidad familiar. Crecen cuando aumenta

2 Por supuesto, los inconvenientes crecen cuando se trata de aplicar el enfoque en la comparación entre sociedades o épocas históricas diferentes (Mitterauer y Sieder, 1989). 
la variedad de las conductas relativas al apareamiento y la procreación, y disminuyen siempre que se pueda suponer una cierta uniformidad en lo que a comportamientos nupciales y estructuras familiares se refiere. En las sociedades en las que el apareamiento está fuertemente institucionalizado, la regla de residencia marital es la neolocalidad y la bastardía no se presenta con mucha frecuencia - es decir, allí donde las estructuras de la familia nuclear son predominantes-, la aplicación del enfoque del ciclo vital resulta muy fructífera. La sociedad española ha cumplido tradicionalmente cada uno de esos requisitos en muy aceptable medida, razón por la cual el enfoque del ciclo vital ha sido desarrollado entre nosotros con notable provecho. Concretamente, Del Campo ha presentado en una ya larga serie de publicaciones (véanse, por ejemplo, Del Campo, 1982; Del Campo y Navarro, 1985; Del Campo, 1993) los resultados de una aplicación del enfoque del ciclo vital a la familia española que permite establecer las líneas maestras de su evolución secular.

El objeto de este trabajo es analizar cómo se ha producido en España uno de los, al parecer, inevitables correlatos del proceso de la transición demográfica, a saber: las alteraciones del calendario reproductivo de las mujeres que acompañan a la caída de la fecundidad y que modifican algunas de las magnitudes básicas del ciclo vital de las familias españolas. El sentido de esos cambios es previsible y, en cierto modo, también conocido. De una parte, desde comienzos de los años ochenta se tienen noticias de la evolución descendente de la fecundidad (Aguero y Olano, 1982a y 1982b) que, por así decir, ha situado a la sociedad española en la cresta de la ola antinatalista que recorre algunas sociedades industriales avanzadas (Delgado y Livi-Bacci, 1992). De otra, los trabajos de Del Campo concluyen que la contrastada disminución del número de hijos de los españoles se acompaña de un cierto adelanto en la edad a la maternidad respecto al matrimonio - en el sentido de una reducción del tiempo de espera entre la boda y la llegada del primer vástago- y de una notable y persistente contracción del intervalo fecundo. Hasta donde se alcanza a inferir de lo publicado, estos trabajos acometen la reconstrucción del ciclo vital de la familia española mediante el análisis transversal de la información acopiada en los censos de población. También Delgado (1993) ha abordado el análisis del timing de la fecundidad a partir de las estadísticas vitales, dedicando especial atención a la evolución de los rangos de nacimiento y las edades a la maternidad. Asimismo, el Centre d'Estudis Demografics de Barcelona ha llevado a cabo, bajo la dirección de Anna Cabré, un preciso estudio del calendario de la fecundidad con datos procedentes de los censos y las estadísticas del movimiento natural de la población (Alberdi, 1995: 91 y ss.). Los resultados de estas investigaciones ponen de manifiesto que el largo proceso de rejuvenecimiento del calendario de la maternidad se interrumpe a finales de la década de los años setenta, momento a partir del cual se invierte esa pauta y comienza a elevarse la edad de las madres españolas en consonancia con la disminución de la fecundidad en las edades más jóvenes. Por mi parte, seguiré aquí la metodología propuesta por Feichtinger (1987) y ensayaré una nueva reconstrucción de 
los cambios que ha experimentado el calendario reproductivo de las mujeres españolas, combinando el análisis transversal y el longitudinal a partir de los datos de la Encuesta Sociodemográfica.

\section{FUENTE DE DATOS}

La información sobre la que me baso procede de la Encuesta Sociodemográfica (en adelante, ESD), una fuente estadística de carácter singular ideada para complementar los datos producidos por la última operación censal. Realizada durante el cuarto trimestre de 1991 por el Instituto Nacional de Estadística (INE) a una muestra de casi ciento sesenta mil individuos mayores de diez años y residentes en hogares, la ESD tenía por objeto validar la calidad de los resultados del último censo de población y detectar las dinámicas biográficas que los habían producido. Aunque su muestra se limita a los mayores de diez años, recoge información muy completa de individuos no integrantes de la misma mediante las preguntas que se formulan sobre los parientes más próximos (padres, hijos, hermanos, cónyuges o parejas). Pese a los reconocidos inconvenientes que en estos casos presentan los dispositivos retrospectivos de recogida de información, dicha encuesta constituye una fuente muy adecuada para los fines propuestos, al menos por tres tipos de razones. En primer lugar, porque contiene información abundante no sólo acerca de las características sociodemográficas de la población española, sino también sobre sus trayectorias reproductivas, incluyendo las edades a las que padres y madres tuvieron cada uno de sus hijos; en segundo lugar, porque su amplia muestra minimiza el riesgo de error cuando hay que operar con categorías de frecuencia reducida; y, por último, porque la operación de recogida de datos se sometió a una meticulosa supervisión que ha debido producir resultados más que aceptables.

Bajo tan prometedoras premisas se ha acometido la explotación estadística de los ficheros originales de datos de la ESD con la idea de obtener las distribuciones que permiten medir la duración de la etapa fecunda del ciclo vital y, en último término, analizar el cambio de calendario reproductivo de las españolas. Tales distribuciones son, básicamente, las siguientes (Feichtinger, 1987): a) las tasas específicas de fecundidad para cada edad de la madre según el orden de nacimiento de los hijos, lo que se consigue tabulando las edades de las madres al nacimiento de los hijos de diferentes órdenes ${ }^{3}$; b) la distribución de mujeres para cada edad según su fecundidad acumulada (lo que los anglosajones denominan parity), que es producto de la matriz de probabilidades de

Llegados a este punto, tal vez sea interesante advertir que en el fichero original de datos de la ESD que suministra el Instituto Nacional de Estadística, la información referida a los hijos se encuentra en las fichas 42 a 57, pero éstas no aparecen ordenadas por edad. El procedimiento adoptado para salvar este obstáculo ha consistido en transformar informáticamente los ficheros originales de la ESD, trasponiendo la ubicación de los hijos para que queden colocados por orden cronológico. 
transición de un orden de nacimiento al siguiente para cada edad de las madres: y c) las ratios de progresión en la fecundidad acumulada para cada edad, es decir, la proporción de mujeres con una fecundidad dada que han progresado hasta el hijo siguiente durante una determinada edad. Queda claro, por tanto, que la información fundamental para llevar a cabo los cálculos propuestos es la referente a la fecundidad (número de hijos) por edad de la madre y orden de nacimiento de los hijos, a partir de la cual, y siguiendo los pasos que se explicitarán más abajo, se obtienen las mencionadas distribuciones.

Como también se comprobará más adelante, una vez que se cuenta con esas distribuciones se pueden calcular fácilmente no sólo las tasas específicas de fecundidad para cada orden de nacimiento de los hijos y edad de las madres, sino también las probabilidades de nacimiento de los diferentes órdenes de hijos, así como las edades medias de las madres al nacimiento de los hijos en función de su orden. Adoptando los supuestos de la cohorte sintética, el análisis transversal permite obtener una medida resumen del comportamiento fecundo de todas las generaciones copresentes en la encuesta, es decir, del tempo de la fecundidad de las españolas en un determinado momento. Si, por otra parte, esas mismas operaciones se hacen controlando la cantidad de hijos habidos (y, de nuevo, según su orden de nacimiento), es posible precisar las edades medias de nacimiento de los hijos de cada orden para cada nivel de descendencia acumulada; ello permite, a su vez, determinar los intervalos medios entre nacimientos de distinto orden y también la duración del intervalo fecundo. Pero, naturalmente, estos últimos cálculos sólo tienen sentido para las generaciones de mujeres que ya han concluido su ciclo reproductivo, con lo que este tipo de ejercicio sólo se puede practicar cabalmente desde la perspectiva longitudinal del análisis de la descendencia de las cohortes.

Puesto que el interés fundamental de este trabajo se centra en obtener ciertas medidas del calendario reproductivo femenino, es necesario precisar que se han adoptado dos convenciones. Por lo que se refiere a la primera, en el cálculo de las edades de las madres sólo se han tenido en cuenta los hijos consanguíneos o carnales, pero no los hijastros ni tampoco los hijos adoptados. Esta precisión, que puede parecer una mera perogrullada cuando de lo que se trata es de calcular las edades a la maternidad, no lo es tanto si se tiene en cuenta que lo que en realidad interesa es el calendario reproductivo, y que las tareas reproductivas deberían entenderse en un sentido laxo (Robertson, 1991), en unos términos que desbordan ampliamente las obligaciones domésticas que de forma inmediata se derivan de los partos. En todo caso, el análisis se restringirá aquí al calendario reproductivo en sentido estricto, es decir, al de la maternidad biológica. En cuanto a la segunda de las convenciones adoptadas, los partos múltiples se han considerarado, como también es habitual en este tipo de análisis, únicos a efectos de cómputos de tiempo. Aunque no hay duda de que los partos múltiples (entre un 1,5 y un 2 por 100 de todos los partos) intensifican las labores de la reproducción para las mujeres que los experimentan, son perfectamente equivalentes a los simples en el cálculo del calendario de la maternidad. 
Es momento ahora de mencionar también algunas de las limitaciones que se derivan de la propia naturaleza de la fuente de datos elegida ${ }^{4}$. La primera de esas limitaciones tiene que ver con el hecho de que la encuesta se hace exclusivamente a individuos que residen en hogares, y excluye por lo tanto a aquellos que viven en establecimientos colectivos como residencias de ancianos, conventos, prisiones, hoteles, colegios mayores o cuarteles. Según el Censo de 1991, menos del 1 por 100 de las mujeres españolas vivía en ese tipo de establecimientos, pero las proporciones crecen con la edad hasta alcanzar el 2 por 100 para la población femenina de entre 70 y 74 años y el 5 por 100 para la que entonces tenía más de 75 años. Con toda probabilidad, haber permanecido célibe y no haber tenido hijos facilita a edades avanzadas el internamiento en residencias; de ser éste el caso, la ESD sobreestimaría la fecundidad de las generaciones más viejas, ya que sería mayor la presencia en su muestra de mujeres no solteras y con hijos. En segundo lugar, como sucede siempre que se recurre a un dispositivo retrospectivo de recogida de información como es la encuesta —en la que la reconstrucción de las trayectorias biográficas se confía a los recuerdos del informante-, es poco menos que imposible descartar ciertos errores de medida. Resulta en verdad muy aventurado considerar que tales errores son aleatorios a poco que se presuma una asociación negativa entre la edad y la capacidad memorística de los entrevistados. Parece así más razonable pensar que dicha asociación puede convertirse en una fuente de sesgo, acaso reducida en el caso de la datación de la edad de los hijos por mor del impacto físico y la trascendencia vital que para las mujeres tiene siempre el acontecimiento de la maternidad. Una tercera limitación, que también hay que considerar, procede del hecho de que los datos de encuesta describen fielmente la biografía reproductiva de sus entrevistadas si, y sólo si, la mortalidad y las migraciones no son fenómenos selectivos respecto de la propia reproducción. Como es de suponer, que se cumpla este supuesto resulta problemático. Aunque es posible que para las mujeres más jóvenes no haya (o apenas haya) mortalidad o migración diferencial en función de si han tenido o no hijos, o de su número, de las cohortes de más edad que aparecen en la muestra (las generaciones de mujeres nacidas a comienzos de siglo) es arriesgado conjeturar una mortalidad constante para los diferentes estados civiles — la mortalidad de las solteras puede haber sido mayor que la de las casadas- y, sobre todo, para cada orden de descendencia — pues es seguro que la exposición reiterada al parto potencia la mortalidad- Si a todo ello se añade el hecho de que no pocas de las biografías consideradas en el análisis longitudinal han padecido una guerra civil, se tendrá una idea proporcionada a la posible magnitud de los errores.

${ }^{4}$ Para las fuentes de error de los «dispositivos retrospectivos» frente a los de «observación continua», véanse las indicaciones de Livi-Bacci (1993: 71-72). 


\section{LA FECUNDIDAD EN LA ENCUESTA SOCIODEMOGRAFICA}

Los problemas derivados de la naturaleza de los datos utilizados aconsejan recurrir a algún tipo de contraste que permita calibrar hasta qué punto se adecúa la imagen de la reproducción que se deduce de la ESD a lo que ya se conoce de ese mismo proceso por otras fuentes. El camino elegido para controlar la fiabilidad de los datos utilizados será así el de comparar las magnitudes básicas de la fecundidad que se deducen de la ESD con las que se obtienen de otras fuentes demográficas. En consonancia con la doble forma de análisis que se va a practicar, se harán dos comparaciones entre otras tantas medidas de la intensidad del fenómeno reproductivo entre las españolas. La primera, de índole transversal, tomará en consideración una medida de momento como es el índice sintético de fencundidad (también llamada índice anual de fecundidad o suma de nacimientos reducidos), que expresa para un determinado año el número medio de hijos por mujer de un colectivo de mujeres que, con mortalidad nula, se comportara a lo largo de su biografía fecunda como lo hacen todas las cohortes femeninas que con sus diferentes niveles reproductivos coexisten ese año; la segunda examinará la intensidad de la fecundidad desde un punto de vista longitudinal, y para ello verificará la descendencia final de diferentes generaciones de españolas medida como número medio de hijos por mujer.

En cuanto a la intensidad momentánea de la fecundidad, la medida sobre la que se va a establecer la comparación es el índice sintético de fecundidad. Las fuentes involucradas en la comparación son la ESD y, de nuevo, las estadísticas vitales, los censos y los padrones municipales en la elaboración realizada por el Centre d'Estudis Demografics de Barcelona (Alberdi, 1995: 93), a las que en principio hay que suponer una mayor proximidad a la realidad. Las magnitudes básicas que se exigen para el cálculo del índice son el número de mujeres de cada edad y los hijos habidos, inmediatamente accesibles en los propios datos de la encuesta. Ahora bien, hay que hacer aquí una importante precisión: el trabajo de campo de la ESD se realizó durante el último trimestre de 1991 y sin que hubiera concluido el año, por lo que no tiene mayor sentido referirse a esos meses como base del contraste. El registro de la fecundidad que hace la ESD es necesariamente incompleto para 1991 y procede por ello recurrir a los datos correspondientes a 1990, que en principio no hay razón para pensar que no reflejan fielmente la fecundidad total del año. Con objeto de establecer las comparaciones con base en 1990, se han tomado desplazadas un año las edades de las mujeres tal y como aparecen en la encuesta, se les ha supuesto una mortalidad nula y se les han asignado los hijos nacidos durante ese período. Los resultados se presentan en el cuadro 1 .

De los datos consignados en el cuadro, cabe señalar en primer lugar que el infrarregistro de nacimientos de la ESD con respecto al MNP es mínimo: casi cuatro mil nacidos, es decir, algo menos de un 1 por 100 de todos los nacimientos habidos ese año (una cifra ligeramente superior a los cuatrocientos 
mil). De la distribución por edad de las madres de esos hijos depende en muy buena medida la magnitud del índice sintético de fecundidad: 1,411 hijos por mujer en la ESD frente a 1,364 en la estimación a partir de las estadísticas vitales. La diferencia se cifra así en 0,047 hijos por mujer, ligeramente superior a un 3 por $100^{5}$. Como se puede apreciar en el cuadro, la ESD infravalora ligeramente la fecundidad de las mujeres de 15 a 19 años y de 40 a 44, pero la sobrevalora en el resto de las edades, sobre todo en las que en mayor medida contribuyen a la tasa general de fecundidad. Es muy posible que esta sobreestimación de la tasa general se deba a una mayor facilidad para encontrar y entrevistar a las mujeres con hijos. En todo caso, las diferencias en las estimaciones por edad (en el cuadro aparecen agregadas en grupos quinquenales, pero se han calculado para cada edad simple) son poco apreciables y, afortunadamente, son mayores en términos relativos en las edades que menos contribuyen a la fecundidad general.

\section{CUADRO 1}

Tasas de fecundidad, año 1990

(Datos absolutos en miles)

\begin{tabular}{|c|c|c|c|c|}
\hline Edad & $\begin{array}{c}\text { Número } \\
\text { de mujeres } \\
\text { ESD }\end{array}$ & $\begin{array}{c}\text { Número } \\
\text { de hijos } \\
\text { ESD }\end{array}$ & $\begin{array}{c}\text { Tasas } \\
\text { de fecundidad } \\
\text { ESD }\end{array}$ & $\begin{array}{c}\text { Tasas } \\
\text { de fecundidad } \\
M N P^{*}\end{array}$ \\
\hline 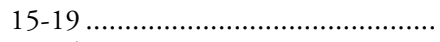 & $1.617,5$ & 11,5 & 0,036 & 0,047 \\
\hline 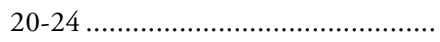 & $1.548,5$ & 75,9 & 0,245 & 0,228 \\
\hline 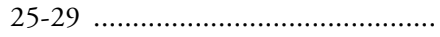 & $1.500,0$ & 152,5 & 0,509 & 0,494 \\
\hline 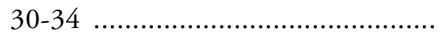 & $1.379,7$ & 117,7 & 0,424 & 0,403 \\
\hline 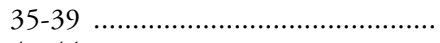 & $1.199,4$ & 39,7 & 0,164 & 0,152 \\
\hline …................................ & $1.184,7$ & 6,4 & 0,027 & 0,035 \\
\hline 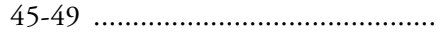 & $1.082,2$ & 1,5 & 0,007 & 0,003 \\
\hline \multirow[t]{2}{*}{ 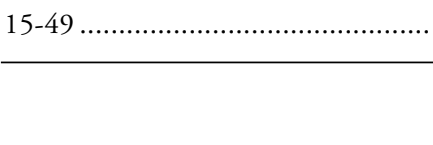 } & $9.512,0$ & 405,2 & 1,411 & 1,364 \\
\hline & & $M N P$ & $E S D$ & $\begin{array}{l}\text { Diferencia } \\
E S D-M N P\end{array}$ \\
\hline Número de nacimientos .................... & (n).............. & 401,4 & 405,2 & $3,8(1,0 \%)$ \\
\hline Número de hijos por mujer (ISF). & & $1,364^{*}$ & 1,411 & $0,047(3,4 \%)$ \\
\hline
\end{tabular}

* Estimación del Centre d'Estudis Demografics (Alberdi, 1995: 93).

FUENTE: Elaboración propia a partir de la Encuesta Sociodemográfica.

5 La estimación del ISF a partir de las estadísticas vitales que ha hecho Delgado (1993) para 1990 es de 1,349 hijos por mujer. En este supuesto, la diferencia sería de 0,062 hijos por mujer a favor de la ESD, ligeramente superior al 4 por 100 . 
Pero, tal y como se ha señalado más arriba, uno de los problemas que plantean los datos de encuesta consiste en la posibilidad de que produzcan distorsiones de la fecundidad de las generaciones de más edad de sus muestras. Tales distorsiones pueden obedecer bien a que la muestra de la encuesta incluye sólo a residentes en hogares (pero no en residencias colectivas), bien a errores derivados de la capacidad memorística de las entrevistadas, bien a pérdida selectiva de universo (y, en consecuencia, también de muestra) - por muerte o migración- en función de su historial reproductivo. Por ello el primer paso consistirá en contrastar los indicadores generacionales que suministran información sobre la fecundidad de las cohortes de más edad, pues constituyen a priori el aspecto más débil de los datos de encuesta sobre biografías reproductivas. En un conocido trabajo, Fernández Cordón (1986) acometió la reconstruccion longitudinal de la fecundidad en España y calculó la descendencia final de las cohortes de españolas nacidas entre 1900 y 1960 a partir de los datos de las estadísticas del movimiento de la población y de los censos recogidos por el INE. El gráfico 1 representa la descendencia final de las generaciones año a año según las estimaciones de Fernández Cordón y de la ESD.

\section{GRAFICO 1}

Evolución de la descendencia final de las generaciones

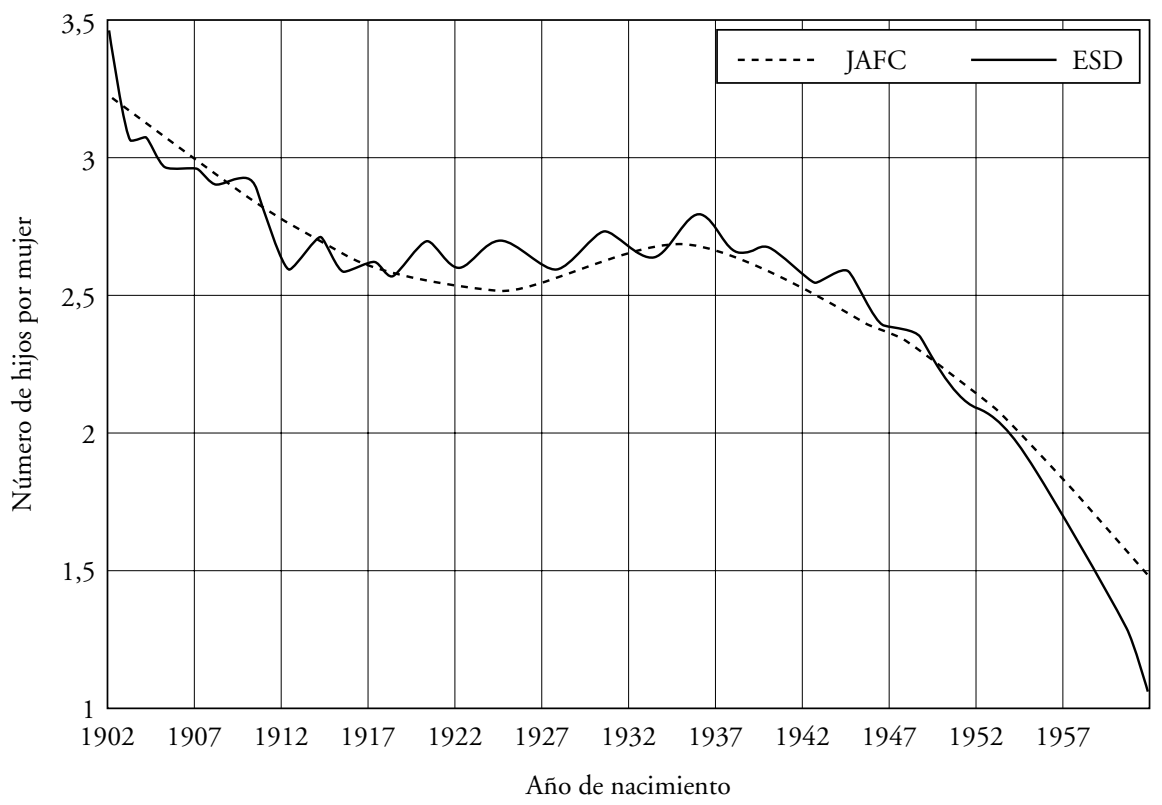

FUENTE: Fernández Cordón (1986) y Encuesta Sociodemográfica (elaboración propia). 
La primera conclusión que se impone a la vista de los datos representados en el gráfico es que la imagen de la evolución secular de la fecundidad de las mujeres españolas no es muy diferente en una y otra fuente. Como se puede comprobar en el mencionado gráfico, el ajuste que proporciona la ESD es bastante aceptable, si bien refleja oscilaciones continuas en torno a la trayectoria mucho más uniforme que refleja la estimación procedente del movimiento natural de la población. Conviene señalar que las oscilaciones son tanto de signo positivo como negativo, aunque en lo que se refiere a las generaciones nacidas durante la primera mitad del siglo los datos de la ESD estiman una fecundidad mayor que las estadísticas vitales para 34 de las primeras 48 cohortes. El fichero de la ESD agrupa a las nacidas antes de 1901 en esa edad, lo que aconseja comenzar la comparación con la cohorte del año siguiente. En este sentido, la mayor discrepancia se produce precisamente en la generación más anciana, la de las mujeres nacidas en 1902-1903, para la que la comparación arroja un saldo positivo en la estimación de la ESD de 0,23 hijos por mujer $(3,46 \text { frente a } 3,22)^{6}$. Para el resto de las cohortes, las diferencias superan la décima sólo en unos pocos años, aunque ambas estimaciones se alejan de nuevo a partir de las generaciones nacidas desde mitad de los años cincuenta, cohortes éstas con las que Fernández Cordón se vio en la obligación de realizar extrapolaciones para lo que en aquel momento constituían datos sobre el futuro.

En síntesis, se puede decir que la imagen que de la biografía reproductiva y los actuales niveles de fecundidad de las españolas transmiten los datos de la ESD es bastante aceptable a juzgar por lo que ya se conoce mediante otras fuentes demográficas. En la mayoría de los casos examinados, las diferencias de las estimaciones que produce la ESD en relación a las estadísticas vitales son superiores en la primera, pero no sobrepasan los márgenes de error comúnmente admitidos cuando se trabaja con encuestas a muestras representativas. Ello es así en lo que se refiere a las medidas transversales sometidas a contraste y a buena parte de las longitudinales, en cuya estimación la ESD se comporta con suficientes garantías de fiabilidad respecto a las estadísticas vitales. El aspecto más problemático se refiere a la descendencia final de las generaciones nacidas en los primeros años del siglo, un colectivo —el de las mujeres que en la fecha de la encuesta tenían cerca de noventa años- para el que incluso la generosa base muestral de la ESD es exigua. Asimismo, hay que señalar que en la reconstrucción de la evolución secular de la descendencia de las generaciones se producen ligeras oscilaciones por encima y debajo de los valores estimados por las estadísticas vitales. Nótese que esos posibles errores de medida, aunque leves en la práctica totalidad de las generaciones, no se distribuyen con arreglo

${ }^{6}$ Como advierte el propio Fernández Cordón, para realizar su estimación se completaron «las generaciones 1901-02 a 1905-06 que carecían de datos iniciales, suponiendo que las tasas que faltaban eran idénticas a la de la misma edad en la generación siguiente. La parte estimada mediante esta sencilla regla supone menos del 4 por 100 en la generación más antigua» (1986: $60)$. 
a una pauta simple o fácilmente discernible. Por ello, la forma de minimizarlos será eminentemente práctica: cuando se practique el análisis longitudinal, se agregarán varias cohortes y se trabajará con generaciones de diez años, de manera que la inevitable pérdida de precisión que se produce al agrupar categorías diferentes se compense con una eventual ganancia de fiabilidad derivada de la reducción de los posibles errores. Una vez agrupadas las cohortes, las diferencias en la descendencia final se sitúan en tres casos (generaciones nacidas entre 1911 y 1920 , entre 1931 y 1940 y entre 1941 y 1950) en el orden de las centésimas; en los dos restantes (mujeres nacidas antes de 1911 y entre 1921 y 1930) superan levemente la décima.

\section{EL CALENDARIO DE LA FECUNDIDAD DESDE LA PERSPECTIVA TRANSVERSAL}

La idea básica para analizar el tempo de la reproducción entre las mujeres españolas consiste en reconstruir sus trayectorias genésicas de modo que quede registrada la intensidad de su fecundidad en cada una de las edades por las que han ido pasando a lo largo de su vida fértil. La observación de la fecundidad que corresponde a cada momento de sus respectivos períodos reproductivos permite calcular una serie de medidas que resumen el calendario de la maternidad, como la edad media al nacimiento de todos los hijos, las edades medias al nacimiento de los hijos de diferente orden, las tasas específicas de fecundidad a las diferentes edades o las tasas específicas de fecundidad de los diferentes órdenes de nacimiento de los hijos. Puesto que esta primera aproximación es de carácter transversal, se han adoptado los supuestos de la cohorte ficticia. Dicho en otras palabras, se ha reconstruido una inexistente generación de mujeres que, no habiendo sufrido ni mortalidad ni emigración selectiva en relación con su capacidad reproductiva, tuviese a sus diferentes edades la misma conducta fecunda que tienen las mujeres efectivamente observadas en el momento de la encuesta. Huelga añadir que sólo en el muy improbable caso de que todas las cohortes copresentes se condujeran de hecho tal y como lo hacen las diferentes edades en el momento de la observación, las pautas transversales registradas en ese preciso momento terminarían reflejando el comportamiento fecundo de una generación auténtica al final de su trayectoria reproductiva. La cohorte ficticia sobre la que se va a trabajar la integran en este caso las mujeres de la muestra que en 1990 tenían más de 14 y menos de 50 años. Representan a 9.511.900 españolas con un número medio de hijos que, como se ha señalado, es de 1,4 por mujer.

El análisis transversal del calendario reproductivo arranca con el examen de las tasas específicas de fecundidad para cada edad de la madre según el orden de nacimiento de los hijos. Dichas tasas se obtienen de tabular, para cada rango de nacimiento, las edades de las madres en el momento en que tuvieron sus hijos nacidos en 1990. Los datos correspondientes se presentan en el cua- 
dro 2, en el que las tasas se expresan en los ya habituales tantos por mil y en el que destaca, en primer lugar, la ya mencionada tasa general de fecundidad (TGF) de 1,4 hijos por mujer. De los resultados registrados en el cuadro, las tasas de fecundidad específicas de cada orden de nacimiento permiten apreciar que el peso que corresponde a los hijos de rango uno y dos se aproxima al 80 por 100 de la fecundidad total. La aportación conjunta de los dos primeros rangos a la tasa general de fecundidad ( 81 por 100 en la ESD) viene a coincidir en lo fundamental con la que se deriva de los datos de momento que suministran las estadísticas vitales (84 por 100), si bien en la estimación de la encuesta el peso de los hijos de primer orden (44 por 100) es ligeramente inferior al de las estadísticas vitales ( 49 por 100), que, en cambio, atribuyen menos peso al segundo hijo que la ESD (Delgado, 1993: 141; Alberdi, 1995: 96). Esa discrepancia se traduce al final en una fecundidad general más alta en la ESD que en las estadísticas vitales. En lo relativo al peso del resto de los órdenes las diferencias son poco significativas, aunque hay que indicar que la base muestral para los órdenes superiores a cinco es, lógicamente, muy pequeña. No hay que perder de vista que estas tasas se refieren al comportamiento de las mujeres de entre 15 y 49 años, y que excluyen por tanto la fecundidad de aquellas que habiendo superado los 50 años (o, para ser exactos, los 51 años en 1991, dado el procedimiento que se ha seguido) ya han dado por finalizado su período reproductivo en el momento de realizarse la encuesta. Merece la pena también reseñar aquí que esas tasas se enmarcan en la conocida evolución de las aportaciones de cada rango de nacimiento a la fecundidad general, en la que los hijos de primer orden y también, aunque en menor medida, los de segundo orden han adquirido un peso cada vez mayor en la fecundidad total, mientras que, como es lógico, lo han ido perdiendo los hijos de tercer y sucesivos órdenes ${ }^{7}$.

El análisis de la fecundidad por edad que se desprende de los datos de la ESD no revela, desde luego, grandes sorpresas. Antes bien, las conclusiones que de esta fuente se pueden obtener acerca del calendario reproductivo de las españolas vienen simplemente a confirmar los datos que suministran las estadísticas vitales. Por lo que se refiere a las tasas específicas por edad de la fecundidad de momento correspondientes al año 1990, se puede apuntar que las edades en las que se concentra la mayor intensidad son las comprendidas entre los 27 y los 32 años, con un pico máximo en los 29. En términos de grupos quinquenales, la fecundidad adquiere una mayor intensidad entre los 25 y los 29 años, aunque es interesante recalcar: a) que las mujeres comprendidas entre los 30 y los 34 años exhiben una fecundidad más intensa que las que tienen entre 20 y 24 años, algo que ya viene sucediendo en España desde la primera mitad de los años ochenta; $b$ ) que la intensidad reproductiva de las mujeres de entre 30 y 34 años (424 hijos por cada mil mujeres) se aproxima a la de las

Véase también el análisis coincidente realizado a partir de las Encuestas de Fecundidad y la Encuesta de Población Activa (Garrido, 1992: 223-225). 


\section{CUADRO 2}

Tasas especificas de fecundidad por orden de nacimiento de los hijos y edad de la madre, 1990

(Tantos por mil)

\begin{tabular}{|c|c|c|c|c|c|c|c|c|c|}
\hline \multirow[b]{2}{*}{ Edad de la madre } & \multicolumn{9}{|c|}{ Orden de nacimiento } \\
\hline & $I$ & $I I$ & $I I I$ & $I V$ & $V$ & $V I$ & $V I I$ & VIII & Total \\
\hline$\ldots \ldots \ldots \ldots \ldots \ldots \ldots$ & 1 & 0 & 0 & 0 & 0 & 0 & 0 & 0 & 1 \\
\hline 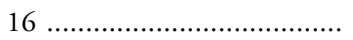 & 2 & 0 & 0 & 0 & 0 & 0 & 0 & 0 & 2 \\
\hline 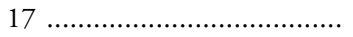 & 8 & 1 & 0 & 0 & 0 & 0 & 0 & 0 & 8 \\
\hline 18 & 5 & 0 & 0 & 0 & 0 & 0 & 0 & 0 & 6 \\
\hline 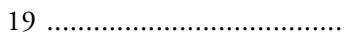 & 13 & 3 & 3 & 0 & 0 & 0 & 0 & 0 & 19 \\
\hline 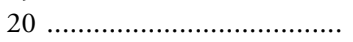 & 22 & 1 & 0 & 0 & 0 & 0 & 0 & 0 & 22 \\
\hline $21 \ldots \ldots \ldots \ldots \ldots$ & 36 & 7 & 1 & 0 & 0 & 0 & 0 & 0 & 44 \\
\hline $22 \ldots \ldots \ldots \ldots \ldots$ & 44 & 11 & 1 & 1 & 0 & 0 & 0 & 0 & 57 \\
\hline 23 & 44 & 13 & 1 & 0 & 0 & 0 & 0 & 0 & 58 \\
\hline $24 \ldots \ldots \ldots \ldots \ldots$ & 44 & 14 & 5 & 1 & 0 & 0 & 0 & 0 & 64 \\
\hline 25 & 53 & 24 & 3 & 0 & 0 & 0 & 0 & 0 & 81 \\
\hline 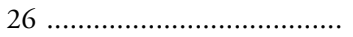 & 54 & 28 & 9 & 2 & 0 & 0 & 0 & 0 & 92 \\
\hline 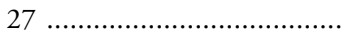 & 67 & 40 & 4 & 1 & 0 & 0 & 0 & 0 & 113 \\
\hline 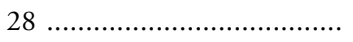 & 53 & 45 & 8 & 1 & 0 & 0 & 0 & 0 & 108 \\
\hline 29 ............................ & 49 & 53 & 11 & 2 & 0 & 0 & 0 & 0 & 116 \\
\hline 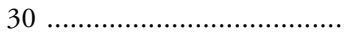 & 36 & 46 & 14 & 6 & 1 & 0 & 0 & 0 & 103 \\
\hline 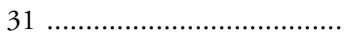 & 24 & 51 & 15 & 2 & 0 & 0 & 0 & 0 & 93 \\
\hline 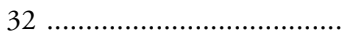 & 23 & 60 & 15 & 5 & 0 & 0 & 0 & 0 & 103 \\
\hline 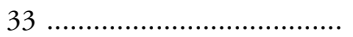 & 8 & 37 & 13 & 3 & 3 & 0 & 0 & 0 & 63 \\
\hline 34 & 13 & 27 & 16 & 5 & 1 & 0 & 0 & 0 & 61 \\
\hline 35 ............................... & 4 & 17 & 15 & 7 & 3 & 0 & 0 & 0 & 46 \\
\hline 36 & 4 & 18 & 10 & 5 & 0 & 2 & 0 & 0 & 38 \\
\hline 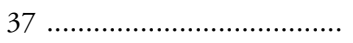 & 4 & 10 & 20 & 8 & 6 & 0 & 0 & 0 & 49 \\
\hline 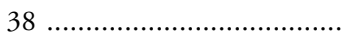 & 2 & 7 & 10 & 0 & 0 & 0 & 2 & 0 & 19 \\
\hline 39 ................................ & 0 & 2 & 4 & 2 & 0 & 2 & 0 & 1 & 12 \\
\hline 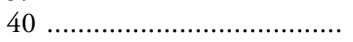 & 1 & 1 & 2 & 3 & 0 & 0 & 0 & 0 & 7 \\
\hline 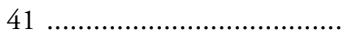 & 2 & 0 & 3 & 1 & 2 & 0 & 0 & 0 & 8 \\
\hline 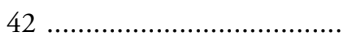 & 1 & 1 & 0 & 2 & 0 & 1 & 0 & 0 & 5 \\
\hline 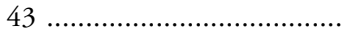 & 0 & 0 & 2 & 1 & 2 & 0 & 0 & 2 & 6 \\
\hline 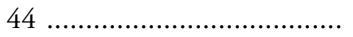 & 0 & 1 & 0 & 0 & 0 & 0 & 0 & 0 & 1 \\
\hline 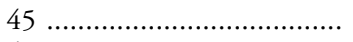 & 0 & 1 & 0 & 3 & 0 & 1 & 0 & 0 & 4 \\
\hline 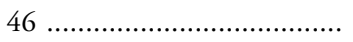 & 0 & 0 & 0 & 0 & 0 & 0 & 0 & 0 & 1 \\
\hline 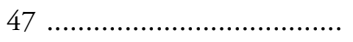 & 0 & 0 & 0 & 0 & 0 & 0 & 1 & 0 & 1 \\
\hline 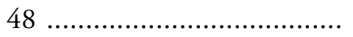 & 0 & 0 & 0 & 0 & 0 & 0 & 0 & 0 & 0 \\
\hline 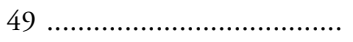 & 0 & 0 & 0 & 0 & 0 & 0 & 0 & 0 & 0 \\
\hline TOTAL …......................... & 618 & 518 & 184 & 61 & 19 & 6 & 3 & 3 & 1.411 \\
\hline$p$ & 0,62 & 0,84 & 0,36 & 0,33 & 0,31 & 0,32 & 0,51 & 0,85 & \\
\hline 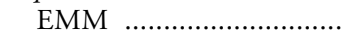 & 26,5 & 30,0 & 32,4 & 34,5 & 36,6 & 39,4 & 41,8 & 41,5 & 29,0 \\
\hline
\end{tabular}

FUENTE: Elaboración propia a partir de la Encuesta Sociodemográfica. 
comprendidas entre los 25 y 29 (509 hijos por mil mujeres); y c) que la fecundidad de las adolescentes, es decir, de las mujeres de menos de 20 años, es muy reducida en la actualidad (menos de 40 nacidos por mil mujeres), prolongándose así una tendencia patente desde 1980. Estos datos constituyen así una prueba indirecta, pues se deduce de un análisis de momento, del proceso de envejecimiento de la maternidad acaecido en los últimos años. En definitiva, con las diferencias antes apuntadas entre la ESD y las estadísticas vitales, el análisis transversal de los datos de la encuesta para el año 1990 permite apreciar el progresivo retraso del calendario reproductivo que se ha producido en los últimos años y que ya han puesto de manifiesto los trabajos referidos ${ }^{8}$.

El análisis del calendario de la fecundidad prosigue con el examen de las edades medias a la maternidad. A partir de las tasas específicas de fecundidad es muy fácil obtener la medida de esas edades medias a la maternidad (EMM), que se han calculado para todos los nacimientos independientemente de su rango y para cada rango particular ${ }^{9}$. Como se puede apreciar, la edad media a la maternidad general es en 1990 de 29 años, coincidente, como se recordará, con la edad modal que presentan las tasas específicas. Este alto valor de la edad media a la que las madres han tenido todos sus hijos en 1990 — muy próximo por lo demás al estimado a partir de las estadísticas vitales: 28,84 ó 28,86 años, según los diferentes cálculos de las autoras reseñadas- no es sino otra forma de calibrar el retraso en el calendario reproductivo que se deduce del análisis de las tasas por edad. Cuando se pone este dato de la edad media a la maternidad en una perspectiva temporal, la sucesión de datos transversales permite apreciar la posposición de la maternidad que se viene experimentando desde comienzos de los años ochenta y que invierte así la documentada tendencia rejuvenecedora de años previos. Por otro lado, al inspeccionar las edades medias a las que se tienen los hijos de los diferentes órdenes — de nuevo muy próximas a las estimadas mediante las estadísticas vitales - es posible constatar también la elevada edad a la que se tiene el primer hijo (26,5 años) y el hecho destacado de que para los hijos de orden dos y superiores las edades medias se encuentran virtualmente por encima de los 30 años.

Partiendo asimismo de las tasas específicas de fecundidad para cada orden de nacimiento se pueden calcular también las diferentes ratios de progresión en los diferentes rangos de fecundidad, es decir, las proporciones de mujeres con

8 Desde el Centre d'Estudis Demografics se afirma que «... desde 1979 hasta $1990 \ldots$ las mujeres menores de 30 años han ido reduciendo su fecundidad total, mientras que más allá de esta edad se experimentaba a partir de 1986 una estabilización e incluso una ligera tendencia al alza en las tasas de fecundidad» (Alberdi, 1995: 99). A la misma conclusión llega Delgado (1993: 142).

9 La fórmula que se ha seguido para el cálculo de la edad media a la maternidad es la siguiente:

$$
\mathrm{EMM}=\Sigma(x+1 / 2) b(x) / \Sigma b(x)
$$

donde $x$ es la edad de la madre y $b$ el número de nacimientos. 
un nivel determinado de fecundidad que en algún momento de su trayectoria han alcanzado el siguiente ${ }^{10}$. Dada la escasa capacidad reproductiva de la sociedad española, no es extraño que las probabilidades de proseguir la vida fecunda se reduzcan de forma poco menos que drástica después del segundo hijo. En el año 1990, sólo 62 de cada cien mujeres potencialmente fértiles tendrían su primer hijo ${ }^{11}$ y algo más de ocho de cada diez mujeres que han tenido ya el primer hijo se decidirían por el segundo. A partir de ahí la probabilidad de seguir produciendo hijos apenas supera el 30 por 100, con el consabido efecto de que la acumulación sucesiva de estas bajas probabilidades a lo largo de los diferentes rangos de hijos se convierte en una tasa general de fecundidad muy reducida ${ }^{12}$.

Este tipo de análisis se completa con la hipotética distribución por edad y descendencia de una cohorte de mujeres que invariablemente siguiera las pautas de fecundidad observadas en el año 1990 (cuadro 3) ${ }^{13}$, así como con las razones de progresión en la fecundidad acumulada que esa misma cohorte sintética tendría a lo largo de su vida reproductiva (cuadro 4) ${ }^{14}$. Las distribuciones

${ }^{10}$ La fórmula para el cálculo de estas razones es:

$$
p_{i}=\mathrm{TGF}_{i+1} / \mathrm{TGF}_{i}
$$

siendo $i$ el orden de nacimiento. La tasa general de fecundidad no es más que la suma de nacimientos (reducidos), es decir:

$$
\mathrm{TGF}=\Sigma b(x)
$$

11 El lector sabrá disculpar la tediosa insistencia en que el análisis se hace desde la perspectiva transversal y en que se trata de datos de momento: de entre esas 38 mujeres de cada cien de la cohorte ficticia que durante el año al que corresponde el análisis no han tenido un hijo de primer orden, algunas ya lo han tenido antes y, por lo tanto, les resulta imposible experimentar en la realidad ese progreso en su fecundidad.

12 Para la comparación con las estadísticas vitales, véase otra vez el trabajo del Centre d'Estudis Demografics (Alberti, 1995: 95), en el que se afirma: «Si las pautas de fecundidad fueran... las de 1990 un 33 por 100 renunciaría a ser madres, algo más de la mitad se detendría después del nacimiento del primer hijo, un 48 por 100 tendría un segundo, y un 15 por 100 tendría un tercero."

${ }^{13}$ La distribución de mujeres según su fecundidad acumulada se calcula mediante la fórmula:

$$
d_{i}(x+1)=d_{i}(x)+b_{i}(x)-d_{i+1}(x)
$$

donde $d$ es el número de mujeres, $x$ la edad de la madre, $b$ el número de nacimientos, e $i$ el orden de nacimiento. Para un cálculo mediante la aplicación sucesiva de las probabilidades de nacimiento específicas para cada edad y orden de nacimiento de los hijos, que conduce a los mismos resultados, véase Feichtinger (1987).

14 Para calcular las razones de progresión en la fecundidad acumulada también se ha seguido el procedimiento propuesto por Feichtinger (1987), de acuerdo con la siguiente fórmula:

$$
p_{i}(x)=\sum_{j \geq i+1} d_{j}(x) / \sum_{j \geq i} d_{j}(x)
$$

para todo $x$ y todo $i$, siendo $x$ la edad de la madre e $i$ el orden de nacimiento de los hijos habidos. 


\section{CUADRO 3}

Distribución de mujeres según fecundidad acumulada y edad de la madre, 1990 (Tantos por mil)

\begin{tabular}{|c|c|c|c|c|c|c|c|c|c|}
\hline \multirow{2}{*}{\multicolumn{2}{|c|}{ Edad de la madre }} & \multicolumn{8}{|c|}{ Orden de nacimiento } \\
\hline & & 0 & $I$ & $I I$ & $I I I$ & $I V$ & $V$ & $V I+$ & Total \\
\hline 15 & 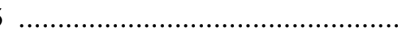 & 1.000 & 0 & 0 & 0 & 0 & 0 & 0 & 1.000 \\
\hline 16 & 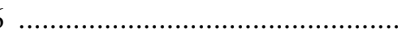 & 999 & 1 & 0 & 0 & 0 & 0 & 0 & 1.000 \\
\hline 17 & (n) & 997 & 3 & 0 & 0 & 0 & 0 & 0 & 1.000 \\
\hline 18 & ….............. & 990 & 10 & 1 & 0 & 0 & 0 & 0 & 1.000 \\
\hline 19 & & 984 & 15 & 1 & 0 & 0 & 0 & 0 & 1.000 \\
\hline 20 & & 971 & 25 & 1 & 3 & 0 & 0 & 0 & 1.000 \\
\hline 21 & & 950 & 46 & 2 & 3 & 0 & 0 & 0 & 1.000 \\
\hline 22 & ................... & 913 & 75 & 8 & 3 & 0 & 0 & 0 & 1.000 \\
\hline 23 & & 869 & 109 & 17 & 4 & 1 & 0 & 0 & 1.000 \\
\hline 24 & & 825 & 140 & 29 & 5 & 1 & 0 & 0 & 1.000 \\
\hline 25 & $\ldots \ldots \ldots$ & 781 & 170 & 38 & 9 & 2 & 0 & 0 & 1.000 \\
\hline 26 & & 728 & 199 & 59 & 12 & 2 & 0 & 0 & 1.000 \\
\hline 27 & & 674 & 224 & 78 & 20 & 4 & 0 & 0 & 1.000 \\
\hline 28 & & 607 & 251 & 115 & 23 & 5 & 0 & 0 & 1.000 \\
\hline 29 & & 554 & 259 & 151 & 30 & 6 & 0 & 0 & 1.000 \\
\hline 30 & & 505 & 256 & 192 & 38 & 8 & 1 & 0 & 1.000 \\
\hline 31 & & 469 & 245 & 225 & 46 & 13 & 2 & 0 & 1.000 \\
\hline 32 & & 445 & 218 & 261 & 58 & 15 & 2 & 0 & 1.000 \\
\hline 33 & & 422 & 182 & 305 & 69 & 20 & 2 & 0 & 1.000 \\
\hline 34 & & 414 & 153 & 329 & 79 & 20 & 5 & 0 & 1.000 \\
\hline 35 & & 401 & 139 & 341 & 90 & 24 & 8 & 0 & 1.000 \\
\hline 36 & & 397 & 126 & 343 & 98 & 27 & 7 & 0 & 1.000 \\
\hline 37 & & 393 & 112 & 351 & 103 & 32 & 12 & 2 & 1.000 \\
\hline 38 & (n) & 389 & 106 & 341 & 115 & 34 & 12 & 2 & 1.000 \\
\hline 39 & 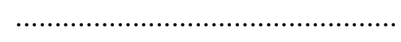 & 387 & 102 & 338 & 125 & 34 & 10 & 2 & 1.000 \\
\hline 40 & & 386 & 100 & 337 & 126 & 36 & 10 & 4 & 1.000 \\
\hline 41 & . & 385 & 100 & 335 & 126 & 39 & 13 & 4 & 1.000 \\
\hline 42 & 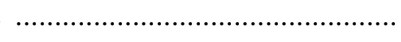 & 383 & 102 & 332 & 128 & 38 & 13 & 4 & 1.000 \\
\hline 43 & & 382 & 102 & 333 & 126 & 39 & 12 & 5 & 1.000 \\
\hline 44 & & 382 & 102 & 332 & 127 & 39 & 13 & 5 & 1.000 \\
\hline 45 & (1) & 382 & 101 & 333 & 127 & 38 & 14 & 5 & 1.000 \\
\hline 46 & 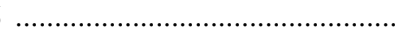 & 382 & 100 & 333 & 124 & 41 & 14 & 5 & 1.000 \\
\hline 47 & (n) & 382 & 100 & 333 & 124 & 41 & 13 & 6 & 1.000 \\
\hline 48 & 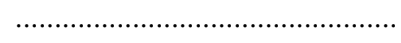 & 382 & 100 & 333 & 124 & 41 & 13 & 6 & 1.000 \\
\hline 49 & 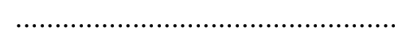 & 382 & 100 & 333 & 124 & 41 & 13 & 6 & 1.000 \\
\hline
\end{tabular}

FUENTE: Elaboración propia a partir de la Encuesta Sociodemográfica. Los procedimientos de cálculo de esta distribución se explicitan en la nota 13. 


\section{CUADRO 4}

Razones de progresión en la fecundidad acumulada por edad de la madre

\begin{tabular}{|c|c|c|c|c|c|c|}
\hline \multirow[b]{2}{*}{ Edad de la madre } & \multicolumn{6}{|c|}{ Orden de nacimiento } \\
\hline & 0 & $I$ & $I I$ & III & $I V$ & $V$ \\
\hline 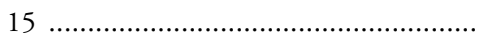 & 0 & 0 & 0 & 0 & 0 & 0 \\
\hline 16 & 1 & 0 & 0 & 0 & 0 & 0 \\
\hline 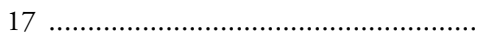 & 3 & 0 & 0 & 0 & 0 & 0 \\
\hline 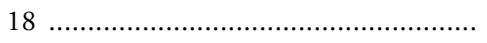 & 10 & 59 & 0 & 0 & 0 & 0 \\
\hline 19 , & 16 & 60 & 0 & 0 & 0 & 0 \\
\hline $20 \ldots \ldots \ldots \ldots \ldots \ldots$ & 29 & 141 & 768 & 0 & 0 & 0 \\
\hline 21 & 50 & 93 & 660 & 0 & 0 & 0 \\
\hline 22 & 87 & 132 & 325 & 82 & 0 & 0 \\
\hline $23 \ldots \ldots \ldots . . .$. & 131 & 169 & 228 & 193 & 0 & 0 \\
\hline 24 . & 175 & 201 & 162 & 171 & 0 & 0 \\
\hline 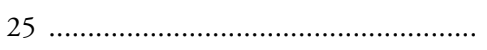 & 219 & 225 & 222 & 179 & 0 & 0 \\
\hline $26 \ldots \ldots \ldots \ldots$ & 272 & 271 & 193 & 138 & 0 & 0 \\
\hline . & 326 & 312 & 228 & 156 & 0 & 0 \\
\hline $28 \ldots \ldots \ldots . . .$. & 393 & 362 & 193 & 168 & 0 & 0 \\
\hline $29 \ldots \ldots \ldots$ & 446 & 418 & 192 & 164 & 55 & 0 \\
\hline $30 \ldots \ldots \ldots$ & 495 & 483 & 196 & 179 & 81 & 0 \\
\hline $31 \ldots \ldots . .$. & 531 & 537 & 212 & 245 & 137 & 166 \\
\hline n. & 555 & 606 & 224 & 229 & 118 & 166 \\
\hline $33 \ldots \ldots \ldots . .$. & 578 & 685 & 229 & 240 & 93 & 166 \\
\hline 34 . & 586 & 739 & 240 & 237 & 198 & 70 \\
\hline 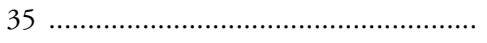 & 599 & 768 & 260 & 246 & 193 & 60 \\
\hline 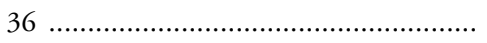 & 603 & 791 & 282 & 268 & 244 & 38 \\
\hline 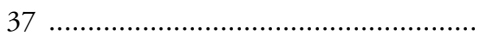 & 607 & 816 & 291 & 284 & 216 & 236 \\
\hline …..................... & 611 & 826 & 325 & 296 & 297 & 139 \\
\hline 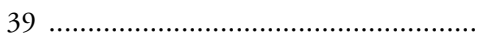 & 613 & 834 & 339 & 279 & 297 & 139 \\
\hline 40 & 614 & 837 & 345 & 286 & 284 & 294 \\
\hline 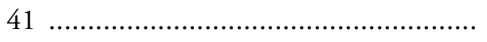 & 615 & 835 & 348 & 297 & 270 & 294 \\
\hline 42 . & 617 & 835 & 355 & 299 & 308 & 252 \\
\hline 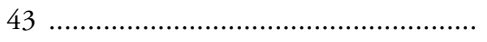 & 618 & 835 & 354 & 308 & 299 & 301 \\
\hline 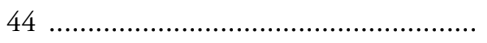 & 618 & 837 & 357 & 309 & 324 & 273 \\
\hline 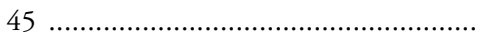 & 618 & 838 & 356 & 309 & 332 & 267 \\
\hline 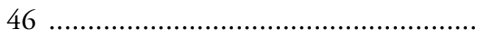 & 618 & 838 & 356 & 328 & 313 & 267 \\
\hline 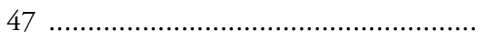 & 618 & 838 & 356 & 328 & 313 & 313 \\
\hline 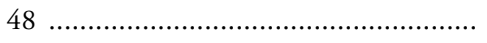 & 618 & 838 & 356 & 328 & 314 & 315 \\
\hline 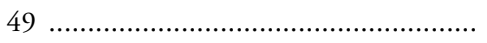 & 618 & 838 & 356 & 328 & 314 & 315 \\
\hline
\end{tabular}

FUENTE: Elaboración propia a partir de la Encuesta Sociodemográfica. Los procedimientos de cálculo de estas razones se explicitan en la nota 14 . 
del cuadro 3 ofrecen una imagen cronológica de la trayectoria reproductiva de esa cohorte ficticia. Si sus pautas de fecundidad permanecieran constantes, esa cohorte se encontraría a los 25 años con menos de una cuarta parte de sus mujeres con hijos, debiendo esperar a después de los 30 años para que la mitad de sus integrantes hubiera tenido alguna descendencia. En este mismo sentido, se puede decir que las probabilidades de pasar de 0 a 1 hijo a los 25 años son del 22 por 100 , mientras que han subido hasta el 50 por 100 a los 30 años (cuadro 4). Puesto que la práctica reproductiva de las adolescentes españolas es en la actualidad muy reducida, antes de los 20 años sólo tendrían algún hijo cinco de cada cien de esas mujeres, entre las cuales muy pocas habrían alumbrado más de uno. Lo que, dicho en términos de probabilidades, significa que a los 20 años sólo tres de cada cien mujeres pasan de no tener hijos a tener el primero. De no variar los comportamientos observados en el año 1990, a la conclusión de su período fértil, casi cuatro de cada diez mujeres no habrían sido madres; de las restantes, una habría tenido un vástago solamente, mientras que algo más de tres habrían visto nacer dos infantes; por último, únicamente dos de cada diez mujeres habrían tenido tres o más hijos.

Por lo tanto, lo que el examen de la fecundidad momentánea observada mediante la ESD pone de manifiesto es — tal y como era de esperar- un modelo de bajo nivel de fecundidad en el que, además, se acorta la fase de la vida dedicada a la reproducción como efecto de su condensación en torno a edades que son cada vez más elevadas cuando comienza el esfuerzo reproductivo y cada vez más jóvenes cuando termina: en los últimos años las mujeres españolas han ido aplazando paulatinamente sus quehaceres reproductivos hasta después de los 25 años y concentrándolos en la treintena. En suma, nos reproducimos cada vez menos y, además, comenzamos a hacerlo cada vez más tarde y terminamos antes. Es sabido que este modelo no sólo responde al cambio estructural hacia un régimen reproductivo que exige una fecundidad de baja intensidad (de hecho, la transición a ese régimen ha permitido un adelanto de calendario de la reproducción durante no pocos años de este siglo), sino también a las ya prolongadas dificultades que los jóvenes españoles experimentan hoy a la hora de integrarse en el mundo adulto. En efecto, cuando la prolongación de los períodos formativos se combina con graves dificultades para incorporarse de forma estable y solvente en el mercado de trabajo y con impedimentos no menos graves y crecientes para procurarse viviendas independientes, las tasas de dependencia familiar de los jóvenes aumentan, las posibilidades de formar parejas y nuevas familias necesariamente disminuyen y la capacidad reproductiva se estanca y pospone hasta edades más elevadas. Tal ha sido la evolución conocida a lo largo de los años ochenta, en la que no sólo ha seguido disminuyendo la intensidad de la fecundidad hasta el punto de situarnos ya muy por debajo del nivel mínimo de reposición, sino que esa disminución se ha realizado desplazando hacia edades más elevadas tanto la puesta en marcha de nuevas empresas familiares como la labor misma de la reproducción. 


\section{EL CALENDARIO REPRODUCTIVO EN LA PERSPECTIVA LONGITUDINAL}

A continuación se abandonará la aproximación transversal y se acometerá el estudio del calendario reproductivo desde una perspectiva estrictamente longitudinal, es decir, siguiendo el comportamiento fecundo de diferentes generaciones (esta vez reales, no sintéticas) de mujeres a lo largo de su trayectoria vital fecunda. Como es obvio, para cada generación el análisis de su calendario reproductivo debe quedar enmarcado en la intensidad de su fecundidad, por lo que esta información se presentará también como característica distintiva que tipifica a cada cohorte. Pero como quiera que los datos referentes a la descendencia final de las cohortes femeninas españolas son ya bien conocidos, y los dispositivos retrospectivos no suelen aportar mayor precisión que los de observación continua, el objetivo primordial consistirá aquí en estimar — siempre por medio de la información acopiada por la ESD - las edades medias a la maternidad de las diferentes cohortes para los distintos órdenes de nacimiento de los hijos. La estimación de esas edades permitirá, a su vez, calcular otro tipo de medidas que resumen muy adecuadamente el calendario de la fecundidad - el intervalo medio entre nacimientos de los hijos de diferente orden, las edades medias al nacimiento del primer y el último hijo y los intervalos fecundos totales de las diferentes cohortes- y que, en consecuencia, definen la duración de fases del ciclo vital de las familias tan fundamentales como son las dedicadas a la reproducción.

Tal y como se indicó más arriba, y por las razones expuestas, las generaciones se compondrán agregando cohortes simples de diez en diez años. Con esa operación se pretende conseguir en cada caso base muestral suficiente para evitar las fluctuaciones asociadas a las pequeñas frecuencias y minimizar los eventuales errores de medida que pudieran afectar, sobre todo, a las mujeres de más edad. En concreto, la solución propuesta hará coincidir las cohortes sometidas a estudio con sucesivas décadas, incluyendo cada una de las generaciones así agregadas a las mujeres nacidas entre el primer y el último año de los cinco primeros decenios del siglo (años terminados en 1 y en 0 ). Puesto que en el caso de los intervalos entre nacimientos el análisis longitudinal sólo tiene sentido para las mujeres que ya han concluido su vida fecunda, se considerarán sólo las mujeres nacidas durante la primera mitad del siglo, advirtiendo de inmediato que la generación de las nacidas entre 1941 y 1950, que tenían en el momento de la encuesta entre 41 y 50 años, no había dado por finalizado su período fecundo y que, por lo tanto, los datos que a ella se refieren no son perfectamente equivalentes a los de las cuatro cohortes decenales que las preceden. No obstante, tomando como punto de referencia la fecundidad instantánea estimada para 1990, se podría decir que a esta generación de las nacidas entre 1941 y 1950 le faltaban 34 hijos por cada mil mujeres para concluir su vida reproductiva activa. Si se han sometido a observación estas cohortes más jóvenes es porque, previsiblemente, esos pocos hijos que les faltan por tener en la 
fase terminal del período fértil no alterarán en mucho las estimaciones que se pueden deducir de las trayectorias fecundas vividas hasta el año 1991.

Las medidas básicas sobre las que se trabajará para analizar longitudinalmente el calendario reproductivo serán las edades medias a la maternidad. Su cómputo obedece al algoritmo ya detallado en el caso del análisis transversal (véase nota 9) a partir de las tasas específicas de fecundidad para cada edad de las madres. La diferencia más reseñable con el procedimiento transversal seguido hasta ahora consiste en que las edades medias a la maternidad se calcularán para cada nivel de descendencia final acumulada. Lo que ahora interesa es determinar las edades medias a las que las madres han alumbrado a sus vástagos dependiendo del número total de hijos que hayan tenido a la finalización de su período fecundo. Ahora bien, ¿por qué se requiere esta desagregación? En principio, los intervalos medios entre nacimientos podrían calcularse como la diferencia entre las edades medias al nacimiento de los rangos consecutivos de hijos, sin que importara el número de hijos habidos. Bastaría entonces con obtener las edades al nacimiento de cada orden de hijos e ir restando unas de otras para obtener una estimación de los distintos intervalos. Finalmente, la diferencia entre la edad media a la que se ha tenido el primer y el último hijo equivaldría al intervalo fecundo medio.

Sin embargo, como previene Feichtinger (loc. cit.: 97), tal proceder sería incorrecto y las estimaciones a que daría lugar sesgadas. El intervalo medio entre dos nacimientos no se puede computar como la diferencia entre la edad media al nacimiento de dos rangos consecutivos de hijos, por la sencilla razón de que para calcular la edad media al nacimiento del hijo de orden inferior se consideran sólo los nacimientos de mujeres que no han tenido ni tendrán nunca otro hijo. Dicho en otros términos, las diferencias entre promedios sucesivos carecen de sentido cuando las medias que se sustraen se obtienen de poblaciones heterogéneas y se refieren a conjuntos diferentes de individuos. El método correcto para calcular los intervalos entre nacimientos consiste más bien en computarlos como la diferencia entre las edades medias al nacimiento de los rangos consecutivos de hijos para aquellas mujeres que han tenido, al menos, un hijo más que el del orden que coincide con el extremo inferior del intervalo que se está calculando ${ }^{15}$. Se entiende así el que para llevar a buen puerto la estimación de los intervalos entre nacimientos se requiera información de la historia reproductiva íntegra de cada mujer y, en particular, las edades medias a la maternidad para cada nivel de descendencia final; fácilmente se

${ }^{15}$ En términos algebraicos, el intervalo medio entre dos nacimientos no se debe calcular como la simple diferencia entre $x_{i+1}$ y $x_{i}$, siendo $x$ la edad media de la madre e $i$ el orden de nacimiento de los hijos, sino más bien como:

$$
t_{i}=x_{i+1}-x_{i}^{i+1}
$$

donde $x_{i}^{i+1}$ representa la edad media de la madre al nacimiento de rango $i$ para las mujeres que han tenido al menos un hijo más que $i$. 
comprende también por qué este tipo de análisis sólo se puede practicar desde la perspectiva longitudinal.

Los procedimientos para computar los intervalos medios entre nacimientos que aseguran resultados no sesgados son así engorrosos, pero no excesivamente complicados. El primer paso consiste en tabular las edades medias a la maternidad para cada orden de nacimientos y cada nivel de descendencia final acumulada. A continuación se calcula la edad media a la maternidad de los diferentes hijos según su orden de nacimiento y también, claro está, la edad media a la maternidad general. Con estos datos (cuadros 5 a 9) se puede ya obtener la edad media a la maternidad según orden de nacimiento de los hijos para todos los niveles de descendencia final, que, como es lógico, no es sino la media ponderada de las medias que corresponden a cada nivel de descendencia final; pero el factor de ponderación - y esto es importante recalcarlo — no es la proporción que cada nivel de descendencia final representa sobre el total de mujeres, sino la proporción que las mujeres con un determinado número de hijos representan sobre el conjunto de sí mismas y superiores niveles de fecundidad. De no hacerse así, se estaría atribuyendo a las madres con un determinado nivel de descendencia un peso que en realidad no les corresponde. Mediante esta ponderación se pretende evitar que las mujeres que se han detenido en un determinado nivel de descendencia entren en el cálculo de la edad media a la que otras madres han tenido los hijos de orden superior. Para el cálculo de la edad a la maternidad de los hijos de un determinado orden sólo se deben tener en cuenta a las mujeres que han alcanzado ese rango u otro más alto. Con otras palabras, el peso que permite calcular correctamente la edad media a la maternidad para los diferentes órdenes de nacimiento es la probabilidad de que una mujer con un determinado número de hijos termine su carrera fecunda con al menos un hijo más ${ }^{16}$.

Tomando parecidas precauciones, pero utilizando diferentes factores de ponderación, se calculan las medidas que tal vez mejor sintetizan el calendario de la reproducción: los intervalos fecundos totales y las edades al nacimiento del primer y el último hijo (cuadro 10). El intervalo fecundo total $\left(\varepsilon_{t}\right)$-es decir, el lapso que media entre el nacimiento de rango uno y el de rango superior - se computa como la media de las diferencias entre los primeros y últimos nacimientos para cada nivel de descendencia final, ponderadas por el peso

${ }^{16}$ Expresado de nuevo en términos algebraicos (Feichtinger, ídem: 97), la edad media a la maternidad según orden de nacimiento se calcula como sigue:

$$
x_{i}=\sum_{j=i}^{m}\left(d_{j} / l_{i}\right) x_{i . j}
$$

donde $x$ es la edad de la madre, $i$ el orden de nacimiento de los hijos habidos, $j$ el número de hijos, $m$ el número máximo de hijos, $d_{j}$ el número de mujeres con $j$ hijos, $l_{i}$ el número de mujeres que han tenido $i$ o más hijos, y $x_{i, j}$ la edad media a la maternidad del $i$-ésimo nacimiento de las mujeres cuya descendencia final es $j$. Paralelamente:

$$
x_{i}^{i+1}=\sum_{j=i+1}^{m}\left(d_{j} / l_{i+1}\right) x_{i . j}
$$




\section{CUADRO 5}

Edades medias a la maternidad según orden de nacimiento de los hijos y descendencia final acumulada e intervalos entre nacimientos de la generación de mujeres nacidas antes de 1911

\begin{tabular}{|c|c|c|c|c|c|c|c|c|c|c|c|c|c|c|c|c|}
\hline \multirow[b]{2}{*}{ Orden de nacimiento } & \multicolumn{14}{|c|}{ Descendencia final } & \multirow{2}{*}{$\begin{array}{c}x_{i} \\
\text { Total }\end{array}$} & \multirow{2}{*}{$\begin{array}{l}\text { Inter- } \\
\text { valos }\end{array}$} \\
\hline & 0 & 1 & 2 & 3 & 4 & 5 & 6 & 7 & 8 & 9 & 10 & 11 & 12 & 13 & & \\
\hline . & & 31,0 & 28,2 & 26,4 & 26,0 & 25,4 & 24,7 & 23,9 & 22,7 & 21,5 & 21,0 & 22,4 & 23,4 & 21,8 & 26,7 & 3,2 \\
\hline II & & & 33,0 & 29,8 & 28,3 & 27,8 & 27,0 & 26,3 & 25,5 & 24,3 & 22,4 & 23,9 & 26,0 & 24,0 & 29,2 & 3,4 \\
\hline III & & & & 34,5 & 32,0 & 30,5 & 29,7 & 28,6 & 27,6 & 26,3 & 24,3 & 26,0 & 27,5 & 26,0 & 31,3 & 3,4 \\
\hline 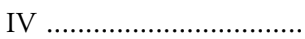 & & & & & 36,5 & 33,8 & 32,5 & 31,0 & 29,7 & 28,5 & 27,5 & 28,0 & 29,3 & 26,7 & 33,4 & 3,1 \\
\hline $\mathrm{V}$ & & & & & & 37,8 & 35,8 & 33,4 & 32,0 & 30,3 & 29,5 & 30,1 & 32,2 & 28,3 & 35,0 & 3,1 \\
\hline VI & & & & & & & 39,7 & 36,4 & 34,5 & 32,5 & 32,0 & 32,2 & 33,7 & 30,2 & 36,6 & 2,7 \\
\hline VII & & & & & & & & 39,3 & 37,3 & 35,2 & 34,0 & 33,9 & 35,2 & 32,0 & 37,2 & 2,7 \\
\hline 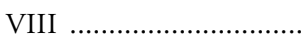 & & & & & & & & & 40,3 & 37,7 & 36,2 & 37,1 & 37,3 & 33,7 & 38,4 & 2,1 \\
\hline 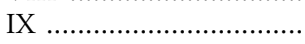 & & & & & & & & & & 39,7 & 38,4 & 39,3 & 39,2 & 36,1 & 39,0 & 2,3 \\
\hline $\mathrm{X}$ & & & & & & & & & & & 40,8 & 41,7 & 40,9 & 37,9 & 40,8 & 2,6 \\
\hline XI & & & & & & & & & & & & 44,6 & 44,1 & 39,3 & 43,5 & 2,5 \\
\hline XII & & & & & & & & & & & & & 44,6 & 42,7 & 43,3 & 3,8 \\
\hline 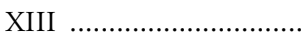 & & & & & & & & & & & & & & 46,5 & 46,5 & \\
\hline 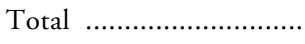 & & & & & & & & & & & & & & & 31,0 & \\
\hline TOTAL MUJERES .......... & 97,4 & 69,4 & 109,4 & 91,7 & 78,2 & 52,2 & 40,1 & 23,8 & 15,8 & 8,4 & 5,2 & 4,3 & 0,6 & 1,3 & 5.978 & \\
\hline \% & 16,3 & 11,6 & 18,3 & 15,3 & 13,1 & 8,7 & 6,7 & 4,0 & 2,6 & 1,4 & 0,9 & 0,7 & 0,1 & 0,2 & 100 & \\
\hline
\end{tabular}

FUENTE: Elaboración propia a partir de la Encuesta Sociodemográfica. Cifras absolutas en miles. 


\section{CUADRO 6}

Edades medias a la maternidad según orden de nacimiento de los hijos y descendencia final acumulada e intervalos entre nacimientos de la generación de mujeres nacidas entre 1911-1920

\begin{tabular}{|c|c|c|c|c|c|c|c|c|c|c|c|c|c|c|c|c|c|c|}
\hline \multirow{2}{*}{$\begin{array}{c}\text { Orden de } \\
\text { nacimiento }\end{array}$} & \multicolumn{16}{|c|}{ Descendencia final } & \multirow{2}{*}{$\begin{array}{c}x_{i} \\
\text { Total }\end{array}$} & \multirow{2}{*}{$\begin{array}{l}\text { Inter- } \\
\text { valos }\end{array}$} \\
\hline & 0 & 1 & 2 & 3 & 4 & 5 & 6 & 7 & 8 & 9 & 10 & 11 & 12 & 13 & 14 & 15 & & \\
\hline I & & 30,4 & 28,3 & 27,1 & 26,1 & 25,7 & 24,9 & 24,0 & 24,0 & 22,2 & 22,8 & 21,7 & 21,3 & 25,5 & 21,0 & 22,5 & 27,3 & 3,5 \\
\hline II ........................... & & & 33,1 & 30,5 & 28,8 & 28,3 & 27,3 & 26,2 & 26,2 & 24,4 & 25,0 & 23,7 & 23,1 & 27,5 & 22,0 & 25,5 & 30,2 & 3,7 \\
\hline III ......................... & & & & 35,3 & 32,6 & 31,2 & 30,0 & 28,8 & 28,3 & 26,5 & 27,1 & 25,3 & 23,9 & 29,5 & 23,0 & 25,5 & 32,5 & 3,6 \\
\hline IV .......................... & & & & & 37,3 & 34,5 & 32,7 & 31,4 & 30,9 & 28,6 & 28,7 & 28,1 & 26,9 & 30,5 & 24,8 & 26,5 & 34,5 & 3,4 \\
\hline V & & & & & & 39,0 & 36,0 & 34,1 & 33,3 & 30,8 & 30,7 & 30,0 & 29,9 & 32,5 & 26,3 & 27,5 & 36,0 & 2,7 \\
\hline VI & & & & & & & 39,5 & 36,7 & 35,7 & 32,4 & 32,4 & 30,5 & 30,6 & 34,5 & 27,8 & 28,5 & 36,8 & 3,0 \\
\hline VII & & & & & & & & 40,6 & 38,6 & 35,0 & 34,0 & 33,2 & 31,9 & 35,5 & 28,8 & 29,5 & 37,8 & 2,8 \\
\hline VIII ….................... & & & & & & & & & 41,8 & 37,6 & 36,5 & 35,9 & 33,9 & 36,5 & 30,8 & 30,5 & 38,9 & 2,5 \\
\hline IX ......................... & & & & & & & & & & 41,0 & 38,9 & 37,6 & 34,7 & 37,5 & 31,8 & 35,5 & 39,0 & 2,7 \\
\hline $\mathrm{X}$ & & & & & & & & & & & 42,0 & 40,1 & 37,1 & 38,5 & 33,5 & 37,5 & 40,3 & 1,9 \\
\hline XI ............................ & & & & & & & & & & & & 41,9 & 39,0 & 39,5 & 36,8 & 39,5 & 40,9 & 2,2 \\
\hline XII ........................... & & & & & & & & & & & & & 41,0 & 41,5 & 39,8 & 41,5 & 40,9 & 2,9 \\
\hline XIII …..................... & & & & & & & & & & & & & & 44,5 & 43,0 & 42,5 & 43,4 & 1,0 \\
\hline XIV …..................... & & & & & & & & & & & & & & & 44,0 & 43,5 & 43,9 & 1,0 \\
\hline XV .......................... & & & & & & & & & & & & & & & & 44,5 & 44,5 & \\
\hline Total ........................ & & & & & & & & & & & & & & & & & 31,3 & \\
\hline TOTAL MUJERES ... & 240,9 & 179,3 & 288,5 & 216,9 & 154,9 & 86,7 & 57,1 & 31,3 & 21,0 & 10,7 & 7,0 & 5,9 & 2,0 & 0,2 & 0,4 & 0,1 & $1.302,9$ & \\
\hline 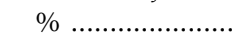 & 18,5 & 13,8 & 22,1 & 16,6 & 11,9 & 6,7 & 4,4 & 2,4 & 1,6 & 0,8 & 0,5 & 0,5 & 0,2 & 0,0 & 0,0 & 0,0 & 100 & \\
\hline
\end{tabular}

FUENTE: Elaboración propia a partir de la Encuesta Sociodemográfica. Cifras absolutas en miles. 


\section{CUADRO 7}

Edades medias a la maternidad según orden de nacimiento de los hijos y descendencia final acumulada e intervalos entre nacimientos de la generación de mujeres nacidas entre 1921-1930

\begin{tabular}{|c|c|c|c|c|c|c|c|c|c|c|c|c|c|c|c|c|c|c|}
\hline \multirow{2}{*}{$\begin{array}{c}\text { Orden de } \\
\text { nacimiento }\end{array}$} & \multicolumn{16}{|c|}{ Descendencia final } & \multirow{2}{*}{$\begin{array}{c}x_{i} \\
\text { Total }\end{array}$} & \multirow{2}{*}{$\begin{array}{l}\text { Inter- } \\
\text { valos }\end{array}$} \\
\hline & 0 & 1 & 2 & 3 & 4 & 5 & 6 & 7 & 8 & 9 & 10 & 11 & 12 & 13 & 14 & 15 & & \\
\hline 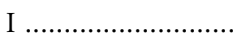 & & 30,8 & 28,3 & 27,1 & 26,1 & 25,5 & 24,4 & 23,9 & 23,2 & 23,2 & 22,5 & 22,0 & 21,5 & 19,7 & 21,2 & 18,3 & 27,4 & 3,6 \\
\hline II & & & 33,3 & 30,5 & 28,7 & 28,0 & 26,9 & 26,2 & 25,1 & 25,2 & 25,3 & 23,8 & 23,5 & 20,3 & 23,5 & 21,9 & 30,4 & 4,0 \\
\hline III $\ldots \ldots \ldots \ldots \ldots \ldots \ldots \ldots$ & & & & 35,6 & 32,6 & 30,8 & 29,5 & 28,4 & 27,3 & 27,4 & 26,9 & 25,3 & 24,9 & 23,5 & 24,6 & 22,9 & 32,8 & 3,7 \\
\hline IV ........................... & & & & & 37,3 & 34,3 & 32,4 & 30,8 & 29,4 & 29,6 & 28,5 & 27,1 & 27,6 & 25,4 & 25,8 & 24,9 & 34,5 & 3,4 \\
\hline $\mathrm{V}$ & & & & & & 38,8 & 35,7 & 32,5 & 31,4 & 31,7 & 30,7 & 28,7 & 29,2 & 26,8 & 27,0 & 30,6 & 35,7 & 3,3 \\
\hline VI & & & & & & & 39,7 & 36,6 & 33,7 & 33,7 & 32,9 & 28,9 & 29,9 & 29,7 & 29,0 & 31,9 & 36,6 & 2,8 \\
\hline VII ...................... & & & & & & & & 40,4 & 36,4 & 35,7 & 34,3 & 31,9 & 32,0 & 31,3 & 30,4 & 33,2 & 36,9 & 2,6 \\
\hline VIII ......................... & & & & & & & & & 39,9 & 37,3 & 37,1 & 34,0 & 34,8 & 34,5 & 32,5 & 34,2 & 37,7 & 2,8 \\
\hline IX ............................. & & & & & & & & & & 40,8 & 39,9 & 36,1 & 35,5 & 36,9 & 34,1 & 35,2 & 39,0 & 2,1 \\
\hline X ............................... & & & & & & & & & & & 42,3 & 37,9 & 38,4 & 39,0 & 35,8 & 36,2 & 39,6 & 2,8 \\
\hline XI & & & & & & & & & & & & 41,9 & 40,1 & 40,7 & 37,1 & 38,2 & 40,4 & 2,3 \\
\hline XII & & & & & & & & & & & & & 42,5 & 43,7 & 39,4 & 39,6 & 41,3 & 2,1 \\
\hline XIII .......................... & & & & & & & & & & & & & & 46,0 & 41,8 & 41,0 & 42,9 & 1,9 \\
\hline XIV …..................... & & & & & & & & & & & & & & & 43,8 & 42,7 & 43,4 & 3,0 \\
\hline 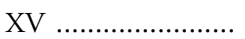 & & & & & & & & & & & & & & & & 45,7 & 45,7 & \\
\hline Total ....................... & & & & & & & & & & & & & & & & & 31,2 & \\
\hline TOTAL MUJERES ... & 309,6 & 256,2 & 519,0 & 381,9 & 238,3 & 130,2 & 76,9 & 33,2 & 24,7 & 17,9 & 8,5 & 5,5 & 1,9 & 1,3 & 1,8 & 1,1 & .008 & \\
\hline \% …....................... & 15,4 & 12,8 & 25,9 & 19,0 & 11,9 & 6,5 & 3,8 & 1,7 & 1,2 & 0,9 & 0,4 & 0,3 & 0,1 & 0,0 & 0,1 & 0,0 & 100 & \\
\hline
\end{tabular}

FUENTE: Elaboración propia a partir de la Encuesta Sociodemográfica. Cifras absolutas en miles. 


\section{CUADRO 8}

Edades medias a la maternidad según orden de nacimiento de los hijos y descendencia final acumulada e intervalos entre nacimientos de la generación de mujeres nacidas entre 1931-1940

\begin{tabular}{|c|c|c|c|c|c|c|c|c|c|c|c|c|c|c|c|c|c|}
\hline \multirow[b]{2}{*}{ Orden de nacimiento } & \multicolumn{15}{|c|}{ Descendencia final } & \multirow{2}{*}{$\begin{array}{c}x_{i} \\
\text { Total }\end{array}$} & \multirow{2}{*}{$\begin{array}{l}\text { Inter } \\
\text { valos }\end{array}$} \\
\hline & 0 & 1 & 2 & 3 & 4 & 5 & 6 & 7 & 8 & 9 & 10 & 11 & 12 & 13 & 14 & & \\
\hline I & & 30,0 & 27,4 & 26,5 & 25,6 & 24,9 & 24,1 & 23,4 & 22,4 & 22,0 & 22,0 & 20,6 & 21,8 & 21,0 & 17,5 & 26,8 & 3,4 \\
\hline II & & & 31,9 & 29,7 & 28,2 & 27,2 & 26,4 & 25,3 & 24,7 & 23,9 & 23,1 & 23,3 & 23,1 & 22,2 & 19,5 & 29,8 & 3,9 \\
\hline III ............................... & & & & 34,6 & 31,8 & 30,1 & 28,7 & 27,3 & 26,8 & 25,9 & 24,1 & 24,7 & 24,1 & 23,5 & 21,5 & 32,3 & 3,7 \\
\hline 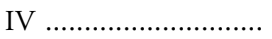 & & & & & 36,4 & 33,4 & 31,4 & 29,7 & 29,0 & 27,6 & 25,6 & 26,9 & 27,0 & 24,8 & 23,5 & 33,9 & 3,5 \\
\hline $\mathrm{V}$ & & & & & & 37,8 & 34,5 & 32,2 & 30,7 & 29,8 & 28,1 & 27,9 & 28,8 & 28,0 & 24,5 & 35,0 & 3,1 \\
\hline 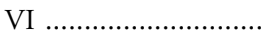 & & & & & & & 38,5 & 35,0 & 32,7 & 31,6 & 31,0 & 28,0 & 29,8 & 29,1 & 26,5 & 35,7 & 2,8 \\
\hline VII & & & & & & & & 38,6 & 35,2 & 33,6 & 33,1 & 31,0 & 31,1 & 30,0 & 27,5 & 35,9 & 3,0 \\
\hline VIII „......................... & & & & & & & & & 38,9 & 36,5 & 35,7 & 32,5 & 33,5 & 31,3 & 28,5 & 36,9 & 3,0 \\
\hline IX & & & & & & & & & & 40,1 & 38,6 & 34,7 & 34,4 & 33,1 & 31,5 & 38,1 & 2,7 \\
\hline $\mathrm{X}$ & & & & & & & & & & & 42,0 & 37,0 & 37,9 & 34,2 & 33,5 & 38,7 & 2,9 \\
\hline 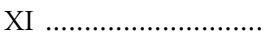 & & & & & & & & & & & & 39,2 & 40,3 & 38,4 & 34,5 & 38,8 & 3,6 \\
\hline 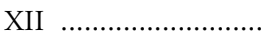 & & & & & & & & & & & & & 43,6 & 42,5 & 35,5 & 42,2 & 1,1 \\
\hline 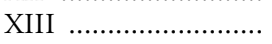 & & & & & & & & & & & & & & 43,5 & 37,5 & 42,7 & 2,0 \\
\hline 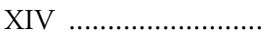 & & & & & & & & & & & & & & & 39,5 & 39,5 & \\
\hline Total .......................... & & & & & & & & & & & & & & & & 30,2 & \\
\hline TOTAL MUJERES ....2 & 27,6 & 229,9 & 647,0 & 490,4 & 270,6 & 129,5 & 72,9 & 33,2 & 19,1 & 11,9 & 5,2 & 2,5 & 1,0 & 2,2 & 0,3 & $2.143,3$ & \\
\hline \% …...................... & 10,6 & 10,7 & 30,2 & 22,9 & 12,6 & 6,0 & 3,4 & 1,6 & 0,9 & 0,6 & 0,2 & 0,1 & 0,1 & 0,1 & 0,0 & 100 & \\
\hline
\end{tabular}

FUENTE: Elaboración propia a partir de la Encuesta Sociodemográfica. Cifras absolutas en miles. 


\section{CUADRO 9}

Edades medias a la maternidad según orden de nacimiento de los hijos y descendencia final acumulada e intervalos entre nacimientos de la generación de mujeres nacidas antes de 1941-1950

\begin{tabular}{|c|c|c|c|c|c|c|c|c|c|c|c|c|c|c|c|}
\hline \multirow[b]{2}{*}{ Orden de nacimiento } & \multicolumn{13}{|c|}{ Descendencia final } & \multirow{2}{*}{$\begin{array}{c}x_{i} \\
\text { Total }\end{array}$} & \multirow{2}{*}{$\begin{array}{l}\text { Inter- } \\
\text { valos }\end{array}$} \\
\hline & 0 & 1 & 2 & 3 & 4 & 5 & 6 & 7 & 8 & 9 & 10 & 11 & 12 & & \\
\hline 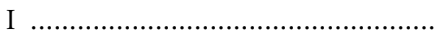 & & 29,3 & 26,3 & 25,0 & 24,0 & 23,3 & 22,8 & 21,8 & 22,2 & 21,6 & 19,2 & 22,4 & 17,5 & 25,7 & 3,1 \\
\hline 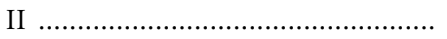 & & & 30,3 & 27,8 & 25,3 & 25,3 & 24,4 & 23,7 & 24,0 & 23,2 & 20,8 & 24,9 & 18,5 & 28,4 & 4,3 \\
\hline III „.................................. & & & & 32,7 & 29,5 & 27,8 & 26,3 & 25,2 & 26,0 & 25,5 & 22,1 & 25,0 & 20,5 & 31,0 & 4,2 \\
\hline 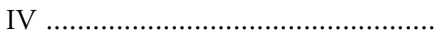 & & & & & 34,6 & 31,1 & 28,7 & 27,3 & 27,7 & 27,4 & 24,0 & 26,3 & 21,5 & 32,6 & 3,8 \\
\hline $\mathrm{V}$ & & & & & & 35,7 & 31,6 & 29,9 & 29,6 & 29,7 & 25,6 & 27,1 & 23,5 & 33,5 & 3,5 \\
\hline VI & & & & & & & 35,9 & 33,6 & 31,7 & 31,4 & 28,0 & 28,5 & 26,5 & 33,9 & 3,2 \\
\hline VII & & & & & & & & 37,6 & 34,0 & 35,0 & 29,9 & 31,5 & 28,5 & 35,1 & 3,5 \\
\hline 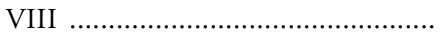 & & & & & & & & & 38,3 & 37,8 & 31,9 & 34,7 & 29,5 & 36,5 & 2,9 \\
\hline IX & & & & & & & & & & 41,5 & 34,6 & 37,1 & 31,5 & 37,0 & 2,9 \\
\hline $\mathrm{X}$ & & & & & & & & & & & 38,6 & 38,8 & 33,5 & 38,2 & 3,6 \\
\hline 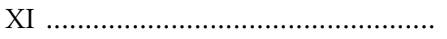 & & & & & & & & & & & & 42,8 & 35,5 & 41,4 & 2,0 \\
\hline XII & & & & & & & & & & & & & 37,5 & 37,5 & \\
\hline Total …............................................ & & & & & & & & & & & & & & 28,5 & \\
\hline 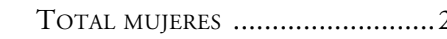 & 220,0 & 225,9 & 847,6 & 569,7 & 236,7 & 98,0 & 34,7 & 15,8 & 10,6 & 2,2 & 2,9 & 2,1 & 0,5 & $2.266,7$ & \\
\hline \% & 9,7 & 10,0 & 37,4 & 25,1 & 10,4 & 4,3 & 1,5 & 0,7 & 0,5 & 0,1 & 0,1 & 0,1 & 0,0 & 100 & \\
\hline
\end{tabular}

FUENTE: Elaboración propia a partir de la Encuesta Sociodemográfica. 
que las mujeres con ese mismo nivel de descendencia representan sobre el total de las mujeres que han alcanzado a tener dos o más hijos ${ }^{17}$. Idéntico factor de ponderación permite calcular las edades medias al nacimiento del primer $\left(\varepsilon_{1}\right)$ y el último hijo $\left(\varepsilon_{u}\right)^{18}$, cuya diferencia es naturalmente el intervalo fecundo. Nótese que las mujeres que no han tenido hijos no se tienen en cuenta para el cálculo del intervalo fecundo; adviértase también que el valor de ese intervalo para las mujeres que han tenido un solo hijo es 0 . Esta última precisión no deja de ser importante, puesto que viene a explicar por qué el valor de esta medida de la edad media al nacimiento del primer hijo $\left(\varepsilon_{1}\right)$, que se refiere a las mujeres que han tenido más de un hijo, no coincide con el valor previamente calculado $\left(x_{1}\right)$, que se refiere a la edad media al nacimiento del primer hijo de todas las mujeres, sea cual fuere su descendencia final.

La información que contienen los cuadros 5 a 9 tipifica la trayectoria reproductiva de las mujeres españolas que han nacido durante la primera mitad de nuestro siglo. La observación de las edades medias a la maternidad, para cada orden de nacimientos de los hijos y para cada nivel de descendencia final, muestra, en primer lugar, la previsible pauta de una disminución casi constante de la edad a la maternidad para cada orden de nacimiento de los hijos a medida que aumenta la descendencia final. Así, por ejemplo, la edad a la que tuvieron su único hijo las mujeres que no han tenido más que uno dista en aproximadamente diez años de promedio de la de las mujeres con diez o más hijos. Parece del todo lógico que las mujeres que terminan su vida fecunda con mayor número de hijos tengan cada uno de ellos a una edad más temprana, aunque sólo sea a causa de los propios costes temporales de la procreación, bien porque la tardanza en dar inicio a la vida reproductiva limite las posibilidades ulteriores de prolongarla, bien porque el calendario reproductivo se adelante en pos de una fecundidad final más intensa. Como quiera que sea, esta importante restricción es respetada prácticamente por todas las mujeres y por todas las generaciones observadas. Unicamente las escasas madres con un número de hijos superior a diez (para las que la base muestral de la ESD es, por lo demás, muy reducida) han conseguido escapar - $\mathrm{y}$ no en todos los casos- a esas severas constricciones temporales que parece imponer la mayor intensidad reproductiva final.

${ }^{17}$ En términos algebraicos, el intervalo fecundo total sería:

$$
\varepsilon_{t}=\sum_{i=1}^{m}\left(d_{i} / l_{1}\right)\left(x_{i . j}-x_{1 . i}\right)
$$

18 En términos algebraicos, la edad al nacimiento del primer hijo sería:

$$
\varepsilon_{1}=\sum_{i=1}^{m}\left(d_{i} / l_{1}\right) x_{1 . i}
$$

mientras la edad al nacimiento del último hijo se calcularía:

$$
\varepsilon_{u}=\sum_{i=1}^{m}\left(d_{i} / l_{1}\right) x_{i . i}
$$

cuando $x_{i . i}$ es el último hijo habido. 
En principio, se podría suponer una relación simple entre la intensidad final y la cronología de la fecundidad femenina en el sentido de que a una menor disposición reproductiva le debe corresponder una mayor condensación del calendario. Así, por ejemplo, los detallados estudios de los historiadores británicos (Anderson, 1990 y 1994) han puesto de manifiesto cómo prácticamente desde que comenzó a reducirse la natalidad a partir de los años treinta del siglo pasado, el período fecundo se concentra en los primeros años del matrimonio. En la experiencia británica, tal condensación no ha sido incompatible con cambios — de no mucha importancia, por cierto- en la edad de nacimiento del primer vástago, pero parece mucho más determinada por el rejuvenecimiento del fin de la etapa fértil. Mientras que la edad del nacimiento del primogénito suele covariar con la edad al matrimonio, que, como se sabe, es muy sensible a la coyuntura económica (Wrigley, 1992), la edad de nacimiento del último hijo parece que ha descendido significativamente desde los momentos en que comenzó a caer la fecundidad. Con toda probabilidad, esa asociación positiva entre caída de la fecundidad y concentración del calendario reproductivo es constante en el largo plazo. No puede ser de otro modo a poco que la reducción de la fecundidad sea una empresa colectiva bien distribuida, ya que la forma de llevarla a la práctica es eliminar los nacimientos de orden superior, que, como se ha señalado, se tienen a edades también superiores.

Sin embargo, las cosas se tornan algo más complejas cuando se observa el cambio desde la perspectiva del corto plazo. Si se consideran ahora las edades a las que las madres españolas han tenido sus diferentes órdenes de hijos, éstas revelan una relación múltiple entre descendencia final y calendario de la reproducción a lo largo de buena parte del siglo (véase gráfico 2). Las mujeres nacidas antes de 1911 y hasta los años treinta experimentaron una indudable disminución de su capacidad reproductiva, que se produjo en la práctica en concordancia con un cierto atraso de la fecha del nacimiento de sus hijos (al menos hasta los de orden ocho). Es patente el cambio de calendario que se produce entre las mujeres de comienzos de siglo y las nacidas entre los años 1911 y 1920, la generación cuya potencialidad reproductiva quedó más dañada por la guerra civil: casi una de cada cinco mujeres de esta generación (18,5 por 100) no tuvo descendencia alguna, la proporción más alta de todas las observadas. Una clara manifestación de esta pérdida es la posposición de la maternidad que se produce entre estas mujeres que vivieron los años más proclives a la procreación en el conflictivo clima social, político y económico que precedió y siguió a la guerra civil ${ }^{19}$. Hay que señalar también que el atraso

19 Como ha señalado Fernández Cordón, la guerra civil «que prácticamente no ha incidido en la descendencia final de las generaciones (los nacimientos impedidos fueron recuperados después) ha provocado, sin embargo, en las generaciones afectadas, una importante alteración del calendario, ya que muchos nacimientos tuvieron que diferirse por la separación de las parejas o por el aplazamiento de los matrimonios... La interpretación es que la Guerra Civil impidió que culminara un proceso de rejuvenecimiento del calendario, que no se reanudaría hasta bastante más tarde» (loc. cit.: 54). 


\section{GRAFICO 2}

Edades medias a la maternidad según orden de nacimiento de los hijos

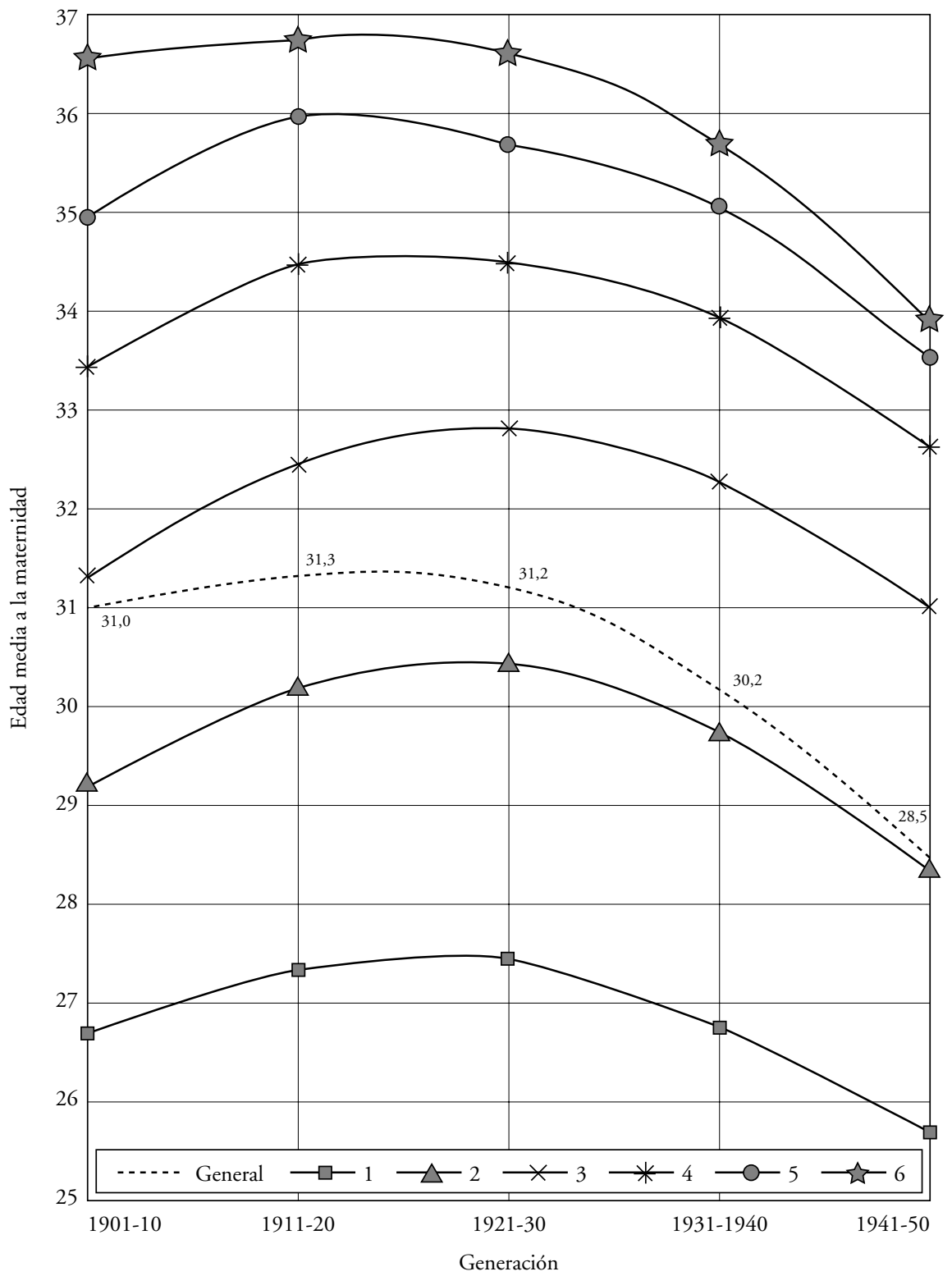

FuENTE: Elaboración propia a partir de los datos de los cuadros 5, 6, 7, 8 y 9. 
parece en este caso muy asociado a la disminución de la fecundidad final, hasta el punto de que entre las generaciones nacidas en los años 1911-1920 y las nacidas en 1921-1930, que han tenido una descendencia virtualmente idéntica (2,65 hijos por mujer), la edad media a la maternidad de los hijos apenas se retrasó (la general es prácticametne idéntica e incluso se adelantó la edad correspondietne a los hijos de orden cinco y superiores, en fechas que corresponden ya a los últimos años cincuenta y primeros sesenta).

El comportamiento reproductivo de las mujeres nacidas en la década de los treinta pone de manifiesto una relación distinta entre calendario e intensidad a la que ejemplificaron las cohortes nacidas durante los primeros años del siglo. En efecto, las nacidas en los años treinta, cuya edad reproductiva se extiende durante los años cincuenta, sesenta y primeros setenta, compatibilizan un ligero aumento de su capacidad reproductiva (hasta 2,70 hijos por mujer) con un notable adelanto del calendario de la fecundidad, con apreciables disminuciones de la edad media a la maternidad en todos los órdenes de hijos. Pero las posibles relaciones entre ambas dimensiones del fenómeno de la reproducción no se agotan ahí, puesto que las mujeres nacidas a lo largo de los años cuarenta han visto cómo disminuía la intensidad de su comportamiento fecundo a la vez que se adelantaba, y de forma notable, su calendario de maternidad. La edad media a la maternidad general de esta generación es de 28,5 años, la más baja de las cinco cohortes estudiadas. Hay que tener en cuenta que la vida fértil de estas mujeres se ha desarrollado en muy buena medida durante dos coyunturas demográficas bien diferentes, pues aunque comienza en los explosivos años sesenta, se extiende también al período recesivo de los años ochenta.

En consecuencia, se puede afirmar que las oscilaciones en la intensidad de la fecundidad que han vivido las primeras generaciones de españolas nacidas en la primera mitad de siglo se han combinado con un proceso general de rejuvenecimiento del calendario de la maternidad, seccionado por las turbulencias que ocasionó la guerra civil. Si se tienen en cuenta ahora los datos analizados desde la perspectiva transversal, se puede afirmar también que ese proceso de rejuvenecimiento se detiene a partir de finales de los años setenta, por lo que es muy probable que, a la conclusión de la vida fértil de las cohortes nacidas después de 1950, se manifieste en los datos longitudinales. Recuérdese a este respecto que la edad media a la maternidad que corresponde a los datos instantáneos de 1990 era ya de 29 años, por encima de la exhibida por las madres nacidas en los años cuarenta.

Estos cambios de calendario reproductivo necesariamente se corresponden con ciertas variaciones en la duración de los intervalos entre nacimientos (gráfico 3). Pues aunque una vida potencialmente fértil amplia y un número no excesivo de hijos permiten a priori no poca diversidad en la distribución temporal de la maternidad ${ }^{20}$, las posibilidades se reducen en la práctica como con-

${ }^{20}$ A pesar de que la duración teórica de la vida fértil se puede estimar en un período inferior a los treinta años, factores de todo tipo hacen que sean extremadamente improbables las situaciones sociales que permiten producir, como promedio, más de cinco o seis hijos por mujer. Incluso en ese caso, no son pocas las posibilidades teóricas de organizar de diferentes formas la cronología de la fecundidad. 


\section{GRAFICO 3}

\section{Intervalos entre nacimientos}

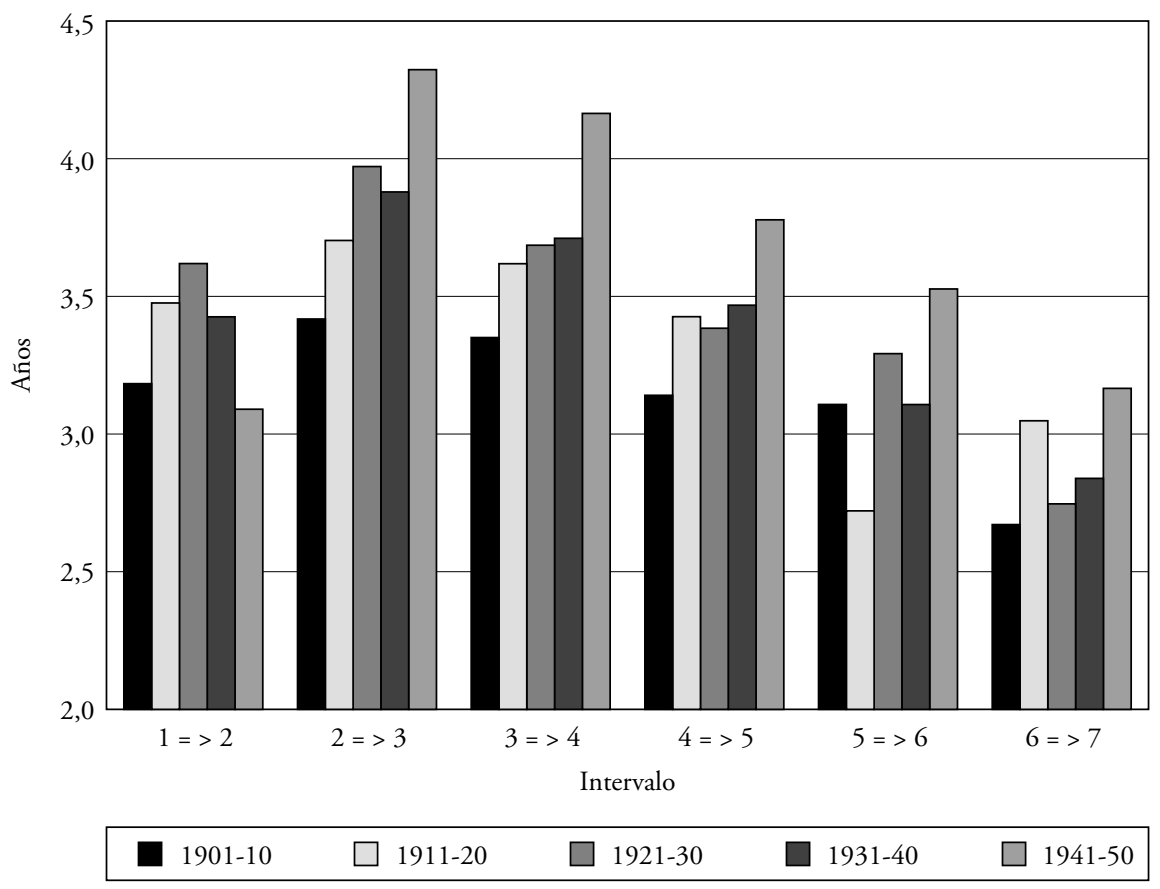

secuencia de las variadas restricciones que siempre pesan sobre el esfuerzo de la reproducción. Así, se puede observar que en términos generales todas las generaciones estudiadas tienden a condensar la cronología de sus esfuerzos reproductivos en los dos primeros hijos - cuyo intervalo es siempre menor que el que se da entre el segundo y el tercero, o entre el tercero y el cuarto- y en los de orden superior a cuatro. Entre las mujeres con mayor descendencia de cualquiera de estas generaciones se vuelve a producir a partir del cuarto hijo un estrechamiento progresivo del margen temporal entre partos, cosa que sucede entre los hijos de orden cuatro, cinco, seis y siete. Ninguna de estas generaciones femeninas ejemplifica mejor esta secuencia vital de concentración reproductiva en el tiempo, dispersión y vuelta a la concentración que la de las nacidas en los años cuarenta. Como tendencia, se puede apuntar también que cada sucesiva generación de mujeres ha ido ampliando, con respecto a la precedente, el tiempo que mediaba entre el nacimiento de sus hijos a partir del segundo. 
Pero si compleja era ya la relación entre intensidad y cronología de la fecundidad, no menos compleja resulta cuando entran en juego los intervalos entre nacimientos. Para empezar, las mujeres nacidas en los tres primeros decenios del siglo — que efectivamente redujeron su fecundidad - tendieron a aumentar de forma progresiva el lapso entre los nacimientos de sus cuatro primeros hijos. En especial, las mujeres más fecundas del siglo son también las que presentan intervalos más cortos en todos los órdenes de nacimientos hasta la entrada en la escena reproductiva de la generación de los años cuarenta. Esta relación negativa entre descendencia final y distancia entre partos se mantiene asimismo entre las nacidas en los años treinta, las cuales - hay que recordarsituaron su capacidad reproductiva por encima de las nacidas en los veinte años anteriores y rejuvenecieron su maternidad, para lo cual tendieron a estrechar el tiempo entre el nacimiento de sus dos primeros hijos. Con todo, estas mismas mujeres consiguieron aumentar su capacidad reproductiva respecto a sus predecesoras de veinte años menos con más distancia entre los partos de orden dos y tres y tres y cuatro. Ahora bien, esto no significa que una caída de la fecundidad se convierta de forma obligada en un mayor espaciamiento de todos los nacimientos. Las mujeres nacidas en los años cuarenta —las de más baja fecundidad y más joven maternidad de entre las observadas - presentan en este sentido un comportamiento muy claro: son aquellas que menos tiempo han dejado transcurrir entre el nacimiento de sus dos primeros hijos, para a continuación pasar a distanciar los nacimientos del resto de su prole en una cuantía no registrada por sus predecesoras. Con una descendencia final de algo más de dos hijos por mujer, el peso de los nacimientos de orden uno y dos viene a ser determinante, y el estrechamiento del intervalo entre los dos primeros nacimientos es responsable del adelanto del calendario reproductivo de esta generación.

Finalmente, el cuadro 10 resume lo esencial de la intensidad y el calendario de la maternidad de esas mismas cohortes. Aunque los datos examinados hasta ahora muestran unas relaciones complejas entre las diferentes dimensiones del fenómeno de la fecundidad cuando se analiza su evolución en detalle, la asociación negativa entre el quantum de la descendencia final y el intervalo de tiempo dedicado a producirla parece bastante clara, incluso si las observaciones se hacen desde la perspectiva del corto plazo. Mientras que las mujeres nacidas antes de 1911 dedicaron más de diez años y dos meses en promedio a engendrar algo más de tres hijos, las nacidas durante los años cuarenta — de las que se puede esperar que su escasa fecundidad pendiente altere en poco las medidas finales de su calendario- redujeron esta fase de sus vidas por debajo de los siete años para alumbrar sólo dos hijos y medio como media. En conjunto, las mujeres españolas han reducido en casi una tercera parte su tiempo reproductivo real en esos cincuenta años; en términos de un simple modelo de regresión bivariable, en el que se incluyen, individuo a individuo, todos los casos pertinentes y se toma el intervalo fecundo como la diferencia simple entre las edades al nacimiento del último y el pri- 


\section{CUADRO 10}

\section{Medidas del calendario reproductivo de diferentes generaciones de mujeres durante el siglo $X X$}

\begin{tabular}{|c|c|c|c|c|c|}
\hline $\begin{array}{c}\text { Fecha de } \\
\text { nacimiento }\end{array}$ & $\begin{array}{c}\text { Descendencia } \\
\text { final }\end{array}$ & $\begin{array}{c}\text { Mujeres } \\
\text { sin hijos } \\
\%\end{array}$ & $\begin{array}{c}\text { Edad media } \\
\text { al nacimiento } \\
\text { del primer hijo } \\
\varepsilon_{1}\end{array}$ & $\begin{array}{c}\text { Edad media } \\
\text { al nacimiento } \\
\text { del último hijo } \\
\varepsilon_{u}\end{array}$ & $\begin{array}{c}\text { Intervalo } \\
\text { fecundo } \\
\text { total } \\
\varepsilon_{t}\end{array}$ \\
\hline Antes $1911 \ldots \ldots \ldots \ldots . .$. & 3,13 & 16,3 & 26,0 & 36,2 & 10,2 \\
\hline 1911-1920 ................ & 2,65 & 18,5 & 26,7 & 36,1 & 9,4 \\
\hline 1921-1930 ............... & 2,65 & 15,4 & 26,8 & 35,9 & 9,1 \\
\hline 1931-1940 ............... & 2,70 & 10,6 & 26,3 & 34,5 & 8,2 \\
\hline 1941-1950 ............... & 2,45 & 9,7 & 25,3 & 32,2 & 6,9 \\
\hline
\end{tabular}

FUENTE: Elaboración propia a partir de la Encuesta Sociodemográfica.

mer hijo ${ }^{21}$, la descendencia final exhibe un considerable nivel de correlación $\left(r^{2}=0,49\right)$ con la amplitud del intervalo fecundo.

Como se puede observar, desde la perspectiva longitudinal la contracción del intervalo fecundo es ininterrumpida a través de las sucesivas cohortes, a un ritmo algo menor a un año de disminución aritmética por cada diez años generacionales. Con todo, hay que subrayar que el proceso de concentración de la fase reproductiva ha sido aún más acusado que el de caída de la fecundidad: si, como se acaba de ver, el intervalo fecundo de las mujeres nacidas en los años cuarenta se ha reducido en casi una tercera parte de su tiempo en relación con la generación de comienzos de siglo, la disminución de las respectivas descendencias finales de esas dos generaciones se puede estimar en poco más de un 20 por 100 . En este sentido, las mujeres de los años veinte ya experimentaron una ligera condensación del calendario manteniendo la misma intensidad reproductiva que sus inmediatas antecesoras; pero mejor ejemplo de este tipo de concentración del ciclo reproductivo lo constituyen las mujeres nacidas en los años treinta, que fueron capaces de engendrar más hijos que las que las habían precedido en los veinte años anteriores en un lapso de tiempo considerablemente menor. Hay que señalar también que la reducción más fuerte del intervalo fecundo parecen experimentarla las cohortes con menos descendencia nacidas en los cuarenta, dato éste que hay que aceptar con las oportunas reservas que pesan sobre una generación cuya fecundidad está todavía por completar.

${ }^{21}$ Cuando se calcula el intervalo fecundo de las mujeres como la diferencia simple entre la edad declarada en la encuesta del año de nacimiento del último y el primer hijo, los intervalos medios para cada generación son los siguientes: 10,29 años (mujeres nacidas antes de 1911); 9,44 (mujeres nacidas entre 1911 y 1920); 9,14 (mujeres nacidas entre 1921 y 1930); 8,17 (mujeres nacidas entre 1931 y 1940); 6,93 (mujeres nacidas entre 1941 y 1950). 
Por otra parte, dada la correlación existente entre la magnitud de la descendencia y el lapso de tiempo dedicado a producirla, la reducción del intervalo fecundo se ha hecho, sobre todo, adelantando la edad a la que se tiene el último hijo, especialmente desde la generación de los años veinte en adelante. Como ya se ha señalado, las españolas atrasaron ligeramente la edad de nacimiento del primer hijo precisamente hasta la generación de las nacidas en los años veinte, en tanto que las generaciones siguientes rejuvenecieron la maternidad, sobre todo las nacidas en los años cuarenta, que han situado la edad del primer parto por debajo de los registros del resto de las generaciones de este siglo. Estas oscilaciones de la edad media del nacimiento del primer hijo contrastan vivamente con el proceso sostenido de disminución de la edad del último parto, que no ha dejado de descender en cada una de las generaciones consideradas (de momento, cuatro años entre la primera y la última, diferencia ésta que con toda probabilidad será al final algo más corta porque la fecundidad pendiente de la última generación terminará por elevar la edad media al nacimiento de su último hijo).

Aunque no tiene una relación directa con el calendario de la reproducción, merece la pena advertir, para terminar, cómo se ha ido reduciendo paulatinamente el número de mujeres sin descendencia desde las generaciones de comienzos de siglo. Si se exceptúa a las mujeres cuya fecundidad resultó más penalizada por la guerra civil — casi una de cada cinco de estas mujeres nacidas entre 1911 y 1920 terminaron su vida fecunda sin descendencia-, la proporción de mujeres sin hijos ha disminuido de forma continua hasta situarse en una cuantía próxima al 10 por 100 entre la generación más joven de las estudiadas, las nacidas en los años cuarenta. El cambio generacional de pautas reproductivas entre las españolas ha provocado así que una proporción creciente de mujeres tuviera con el tiempo un número decreciente de hijos, con lo que ello supone de mayor equidad en la distribución tanto de las cargas como de los beneficios de la reproducción. Si se acepta que el proceso reproductivo está mediado, entre otros factores, por la cuantía de los recursos materiales de los que se dispone, se podría interpretar tentativamente la ampliación progresiva del segmento fecundo de la población femenina como el producto de una distribución cada vez más equilibrada de las posiciones en la estructura social. Ocasión habrá en otro momento de verificar esta hipótesis con la atención que merece.

Por el momento, sólo cabe apuntar que el análisis transversal muestra que ese proceso de apertura progresiva en el acceso a la reproducción ha debido quedar interrumpido como consecuencia de la intensa caída de la fecundidad que ha tenido lugar en los últimos años. Aquí es importante señalar que sólo una de cada cuatro mujeres estudiadas desde la perspectiva transversal forma parte de la última de las cohortes observadas desde la perspectiva longitudinal. Y, lo que es más importante, que entre esas cohortes que en 1990 habían concluido ya su vida fecunda y sus sucesoras se ha producido la «revolución reproductiva» (Garrido, 1996), un proceso que expulsa a proporciones importantes 
de mujeres del sector de la reproducción en el que muchas de ellas han terminado por convertirse en mano de obra excedentaria. Pero hasta que los efectos de esa revolución queden reflejados en la descendencia final de las cohortes nacidas después de 1950, se puede concluir que las mujeres españolas nacidas durante la primera mitad del siglo no sólo han disminuido su fecundidad y han reducido en medida aún mayor el tiempo de sus vidas dedicado a esta empresa, sobre todo por efecto del rejuvenecimiento del calendario de la maternidad y de un adelanto del nacimiento de sus últimos hijos, sino que se han repartido de manera mucho más equitativa las tareas de la reproducción colectiva.

\section{REFERENCIAS BIBLIOGRAFICAS}

AgÜero, Isabel, y Olano, Alberto (1982a): «La evolución reciente de la fecundidad en Espana, en REIS, 10, pp. 121-150.

- (1982b): «La intensa caída reciente de la fecundidad y la nupcialidad en España», en Rosa Conde (comp.), Familia y cambio social en España, Madrid: Centro de Investigaciones Sociológicas, pp. 31-61.

Anderson, Michael (1990): «The social implications of demographic change», en F. M. L. Thompson (ed.), The Cambridge Social History of Britain 1750-1950. Volume 2: People and their environment, Cambridge: Cambridge University Press, pp. 1-70.

- (1994): "What is New about the Modern Family?», en Michael Drake (ed.), Time, Family and Community, Oxford: Blackwell, pp. 67-90.

ARANGO, Joaquín (1987): "La modernización demográfica de la sociedad española», en Jordi Nadal, Albert Carreras y Carles Sudriá (comps.), La economía española en el siglo XX. Una perspectiva histórica, Barcelona: Ariel, pp. 201-236.

ALBERDI, Inés (dir.) (1995): Informe sobre la situación social de la familia en España, Madrid: Ministerio de Asuntos Sociales.

Del CAmpo, Salustiano (1982): La evolución de la familia española en el siglo XX, Madrid: Alianza.

- (1991): La «nueva» familia española, Madrid: Ediciones de la Universidad Complutense.

- (1993): "Fecundidad y familia», en Salustiano del Campo (dir.), Tendencias sociales en España (1960-1990), vol. 1, Bilbao: Fundación BBV, pp. 249-273.

Del Campo, Salustiano, y Navarro, Manuel (1985): Análisis sociológico de la familia española, Barcelona: Ariel.

Delgado, Margarita (1993): "Cambios recientes en el proceso de formación de la familia», en REIS, 64, pp. 123-153.

Delgado, Margarita, y Livi-Bacci, Maximo (1992): «Fertility in Italy and Spain: The Lowest in the World», en Family Planing Perspectives, 24, 4, pp. 162-171.

Feichtinger, Gustav (1987): "The Statistical Measurement of the Family Life Cycle», en John Bongaarts, Thomas K. Burch y Kenneth W. Wachter (comps.), Family Demography. Methods and Their Application, Oxford: Clarendon Press, pp. 81-101.

FERnÁNDEZ CORdón, Juan A. (1986): "Análisis longitudinal de la fecundidad en España», en Alberto Olano (comp.), Tendencias demográficas y planificación económica, Madrid: Ministerio de Economía y Hacienda, pp. 49-75.

GARRIDO, Luis (1992): Las dos biografias de la mujer en España, Madrid: Ministerio de Asuntos Sociales.

- (1996): «La revolución reproductiva», en Cecilia Castaño y Santiago Palacios (eds.), Salud, dinero y amor. Cómo viven las mujeres españolas de hoy, Madrid: Alianza, pp. 205-238. 
Glick, Paul C. (1947): "The Family Cycle», en American Sociological Review, 12, 2, pp. 164-174. GOLDTHORPE, John Ernest (1987): Family life in western societies. A historical sociology of family relationships in Britain and North America, Cambridge: Cambridge University Press.

HöHn, Charlotte (1987): "The Family Cycle: Needed Extensions of the Concept», en John Bongaarts, Thomas K. Burch y Kenneth W. Wachter (comps.), Family Demography. Methods and Their Application, Oxford: Clarendon Press, pp. 65-80.

LIVI-BACCI, Massimo (1993): Introducción a la demografia, Barcelona: Ariel.

Mitterauer, Michael, y Sieder, Reinhard (1989): The European Family. Patriarchy to Patternship from the Middle Age to the Present, Oxford: Basil Blackwell.

RoberTson, A. F. (1991): Beyond the Family. The Social Organization of Human Reproduction, Cambridge: Polity Press.

Tortella, Gabriel (1994): El desarrollo de la España contemporánea. Historia económica de los siglos XIX y XX, Madrid: Alianza.

WRIGLEY, E. Anthony (1992): Gentes, ciudades y riqueza. La transformación de la sociedad tradicional, Barcelona: Crítica.

\section{ABSTRACTS}

This paper sets out to reconstruct some of the basic magnitudes that have defined the lifecycle of the Spanish family throughout this century, and more particularly those relative to the reproductive period. Basic data are drawn from the sociodemographic survey conducted by Spain's National Institute of Statistics (1991) and specifically used with a view to making an indepth analysis of the reproduction tempo of various generations of Spanish women. Basic measurements are related to the age at which women have children and periods of fertility. By conducting a longitudinal study of these data, it is possible to measure the contraction of the genetic period which goes hand in hand with the reduction in the offspring of the generations studied and as regards which the earlier age at which women bear their last child is all-important. Likewise and in order to check the reliability of the source of the data used, a transversal analysis is made of the actual live births that occurred while this study was being conducted. 